\title{
A DFT Approach to the Mechanistic Study of Hydrozone Hydrolysis
}

Author(s): Ibrahim Yildiz*

*Assistant Professor, Applied Mathematics \& Sciences, Khalifa University, PO Box 127788, Abu Dhabi, UAE

Tel: +971 (0)2 401 8208, Fax: +971 (0)2 447 2442, e-mail:ibrahim.yildiz@kustar.ac.ae

\section{Contents}

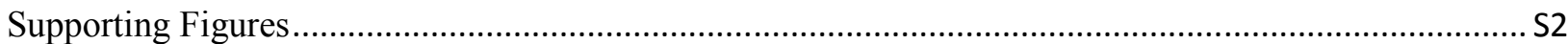

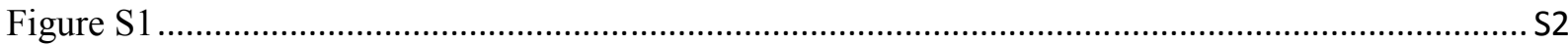

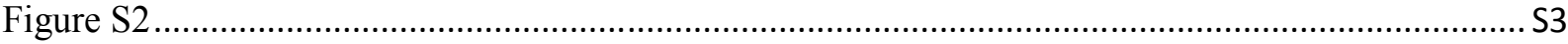

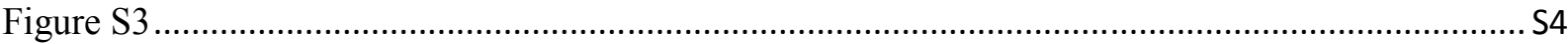

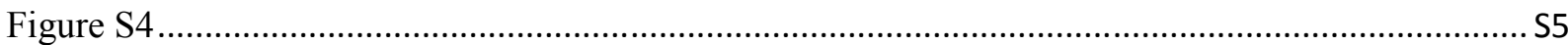

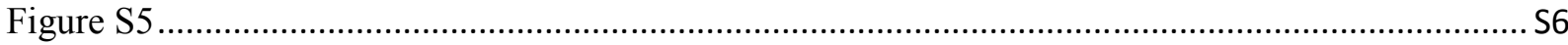

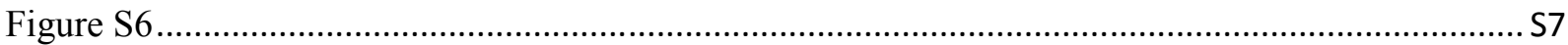

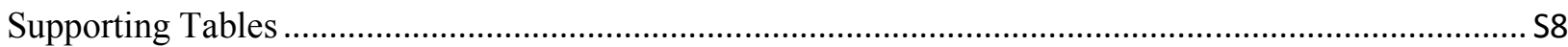

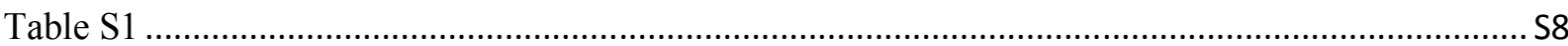

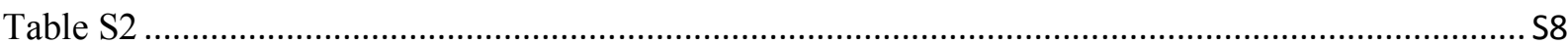

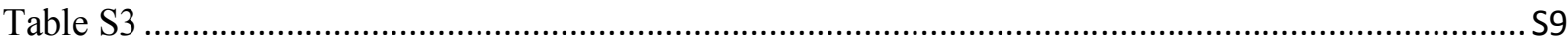

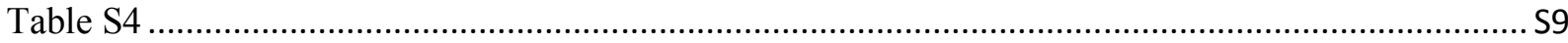

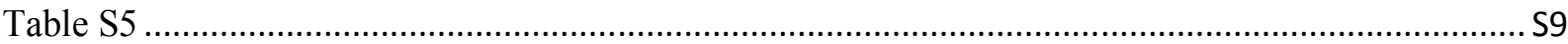

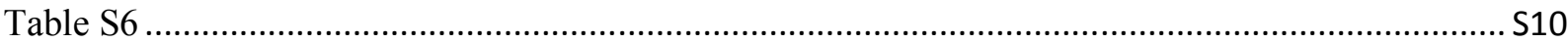

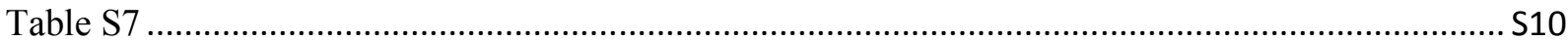

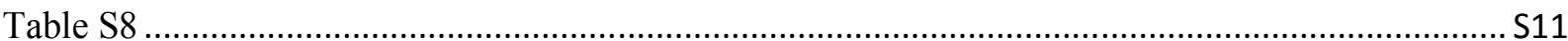

Cartesian Coordinates Corresponding to the Optimized Geometries of All Species involved in Both

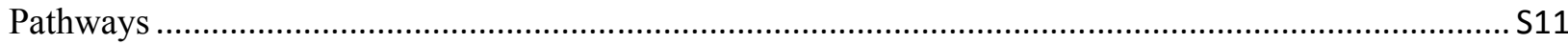

Pathway 1 - One $\mathrm{H}_{2} \mathrm{O}$ Molecule as Reactant ...................................................................................

Pathway 1 - Two $\mathrm{H}_{2} \mathrm{O}$ Molecule as Reactant .............................................................................. 16

Pathway 1 - Three $\mathrm{H}_{2} \mathrm{O}$ Molecules as Reactant............................................................................... 224

Pathway 2 - One $\mathrm{H}_{2} \mathrm{O}$ Molecule as Reactant...................................................................................... 334

Pathway 2 - Two $\mathrm{H}_{2} \mathrm{O}$ Molecules as Reactant.................................................................................. 440

Pathway 2 - Three $\mathrm{H}_{2} \mathrm{O}$ Molecules as Reactant............................................................................... S49 


\section{Supporting Figures}

a)

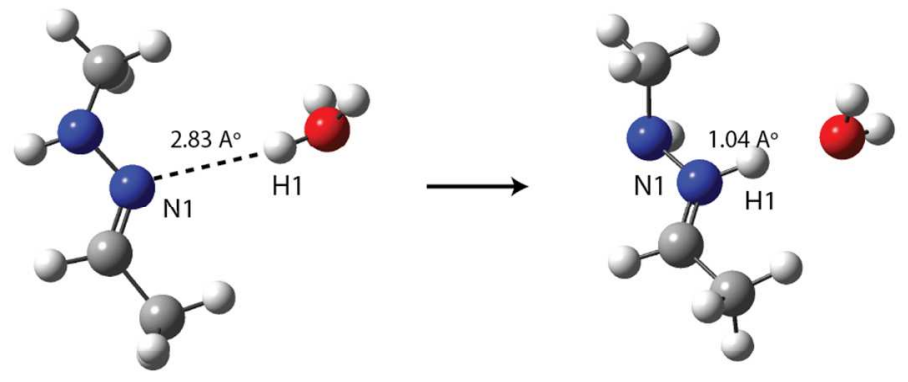

b)

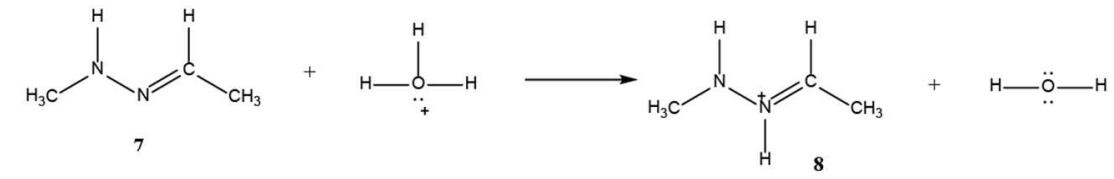

c)

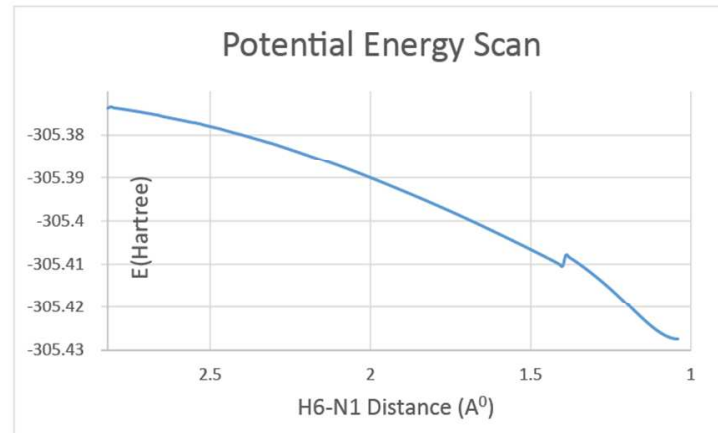

Figure S1. a) The distance between $\mathrm{H}$ atom (H1) in the hydronium ion and $\mathrm{N}$ atom (N1) in model compound 7 was scanned from $2.83 \mathrm{~A}^{\circ}$ to $1.04 \mathrm{~A}^{\circ}$. (Models are the initial and final points in PES) b) Structures of initial and final geometries in PES c) Plot of distance between N1-H1 versus total energy in Hartree 
a)
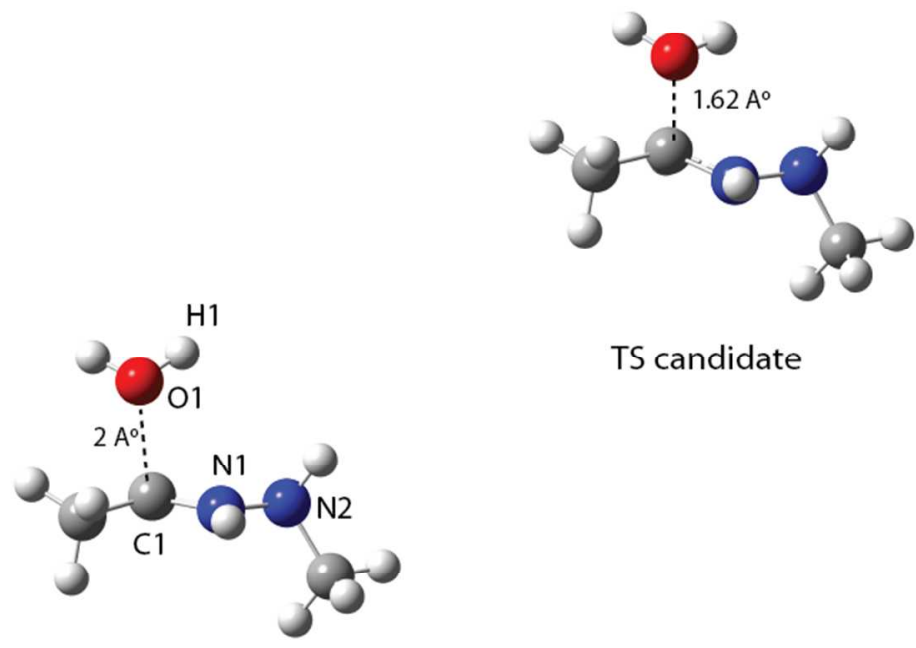

TS candidate

$$
7+\mathrm{H}_{2} \mathrm{O}
$$

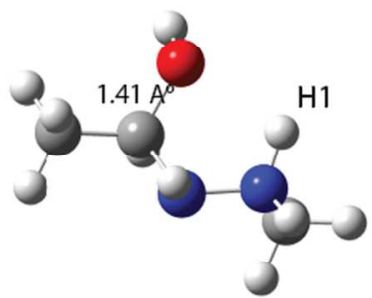

PC

b)

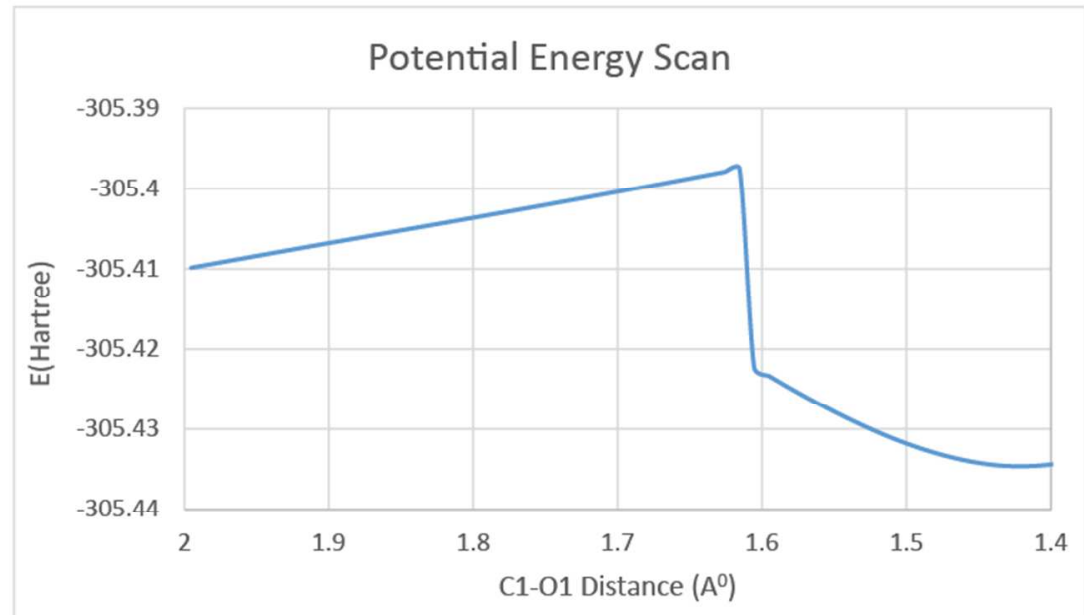

Figure S2. a) The distance between oxygen $(\mathrm{O} 1)$ atom in $\mathrm{H}_{2} \mathrm{O}$ and $\mathrm{C}$ atom $(\mathrm{C} 1)$ in the model compound 8 was scanned from $2.0 \mathrm{~A}^{\circ}$ to $1.40 \mathrm{~A}^{\circ}$ in order to locate a possible TS structure for the water addition step. The initial geometry in the scan was termed as RC (reactant complex), even though it is not an optimized geometry. The highest point in PES was termed as TS (transition state), and the lowest point in PES following TS was termed as PC (product complex). b) Plot of distance between $\mathrm{O} 1-\mathrm{C} 1$ versus total energy in Hartree 
a)

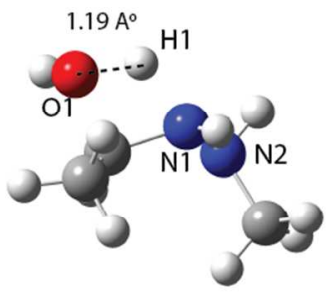

TS candidate

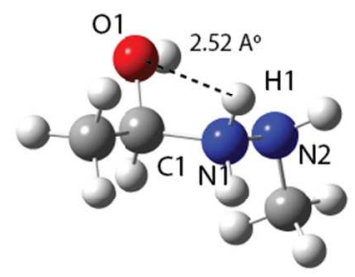

13

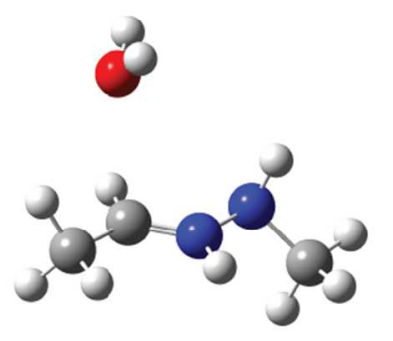

8

b)

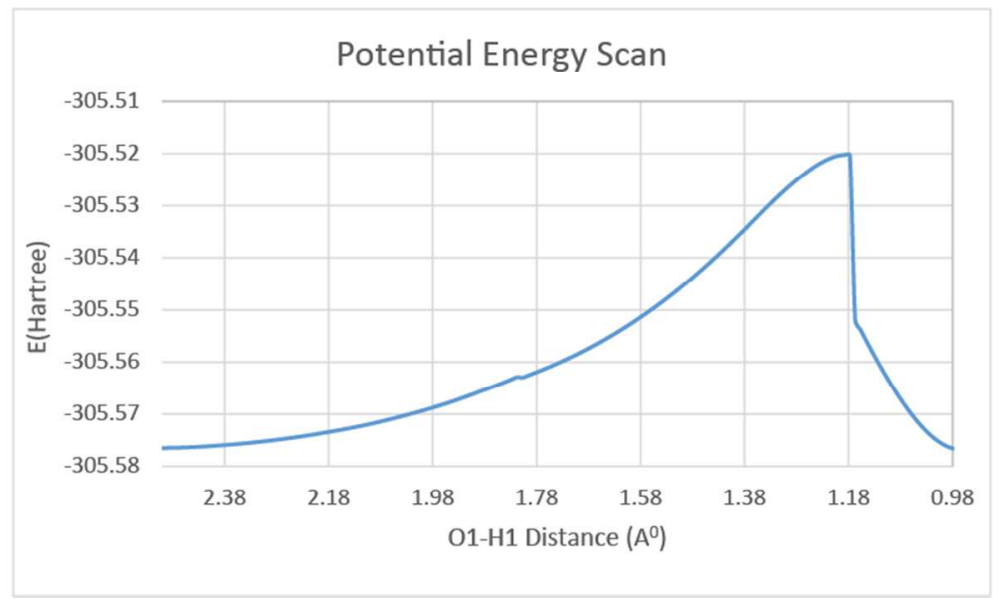

Figure S3. a) The distance between $\mathrm{H}$ atom (H1) connected to $\mathrm{N}$ atom $(\mathrm{N} 1)$ and $\mathrm{O}(\mathrm{O} 1)$ in model compound 13 was scanned from $2.52 \mathrm{~A}^{\circ}$ to $0.90 \mathrm{~A}^{\circ}$. (Models are the initial/final and the highest energy point structures in PES b) Plot of distance between O1-H1 versus total energy in Hartree 


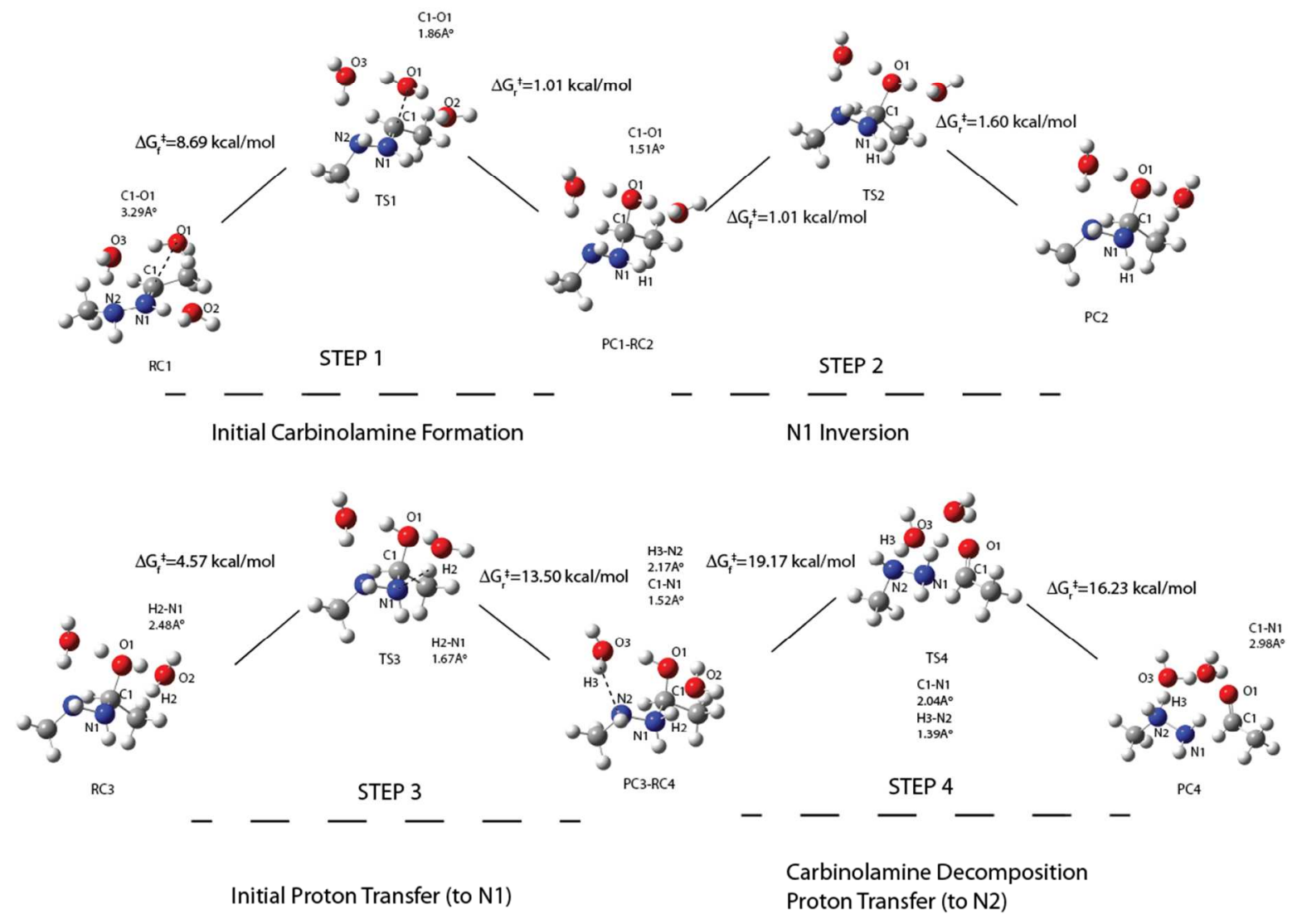

Figure S4. Four-step hydrazone hydrolysis process based on the reaction of three $\mathrm{H}_{2} \mathrm{O}$ molecules with the model compound $\mathbf{8}$ through Pathway 1. In Step 1, C1-O1 bond formation lead to the formation of initial carbinolamine intermediate termed as RC1-PC1 (product complex 1-reactant complex2). After the inversion of N1 (Step 2), an internal proton (H1) transfer occurs to N1 position (Step 3). The resultant carbinolamine decomposes into corresponding products through a second proton (H2) transfer process to N2 (Step 4). 

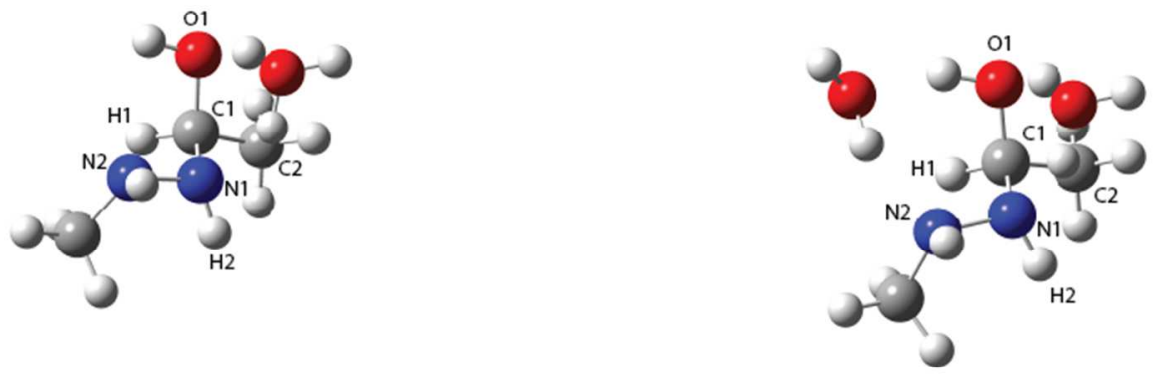

\begin{tabular}{|c|c|c|}
\hline Dihedral & Angle for $2 \mathrm{H}_{2} \mathrm{O}$ & Angle for $3 \mathrm{H}_{2} \mathrm{O}$ \\
\hline O1-C1-N1-H2 & -162.9 & -155.4 \\
\hline $\mathrm{O} 1-\mathrm{C} 1-\mathrm{N} 1-\mathrm{N} 2$ & 73.5 & 81.5 \\
\hline $\mathrm{H} 1-\mathrm{C} 1-\mathrm{N} 1-\mathrm{H} 2$ & 81.1 & 87.7 \\
\hline $\mathrm{H} 1-\mathrm{C} 1-\mathrm{N} 1-\mathrm{N} 2$ & -42.5 & -35.4 \\
\hline $\mathrm{C} 2-\mathrm{C} 1-\mathrm{N} 1-\mathrm{N} 2$ & -168.2 & -159.5 \\
\hline $\mathrm{C} 2-\mathrm{C} 1-\mathrm{N} 1-\mathrm{H} 2$ & -44.6 & -36.3 \\
\hline
\end{tabular}

Figure S5. Dihedral angles around $\mathrm{C} 1-\mathrm{N} 1$ bond for the TS structures corresponding to the proton transfer step to $\mathrm{N} 1$ for Pathway 1 for two and three $\mathrm{H}_{2} \mathrm{O}$ molecule systems. 

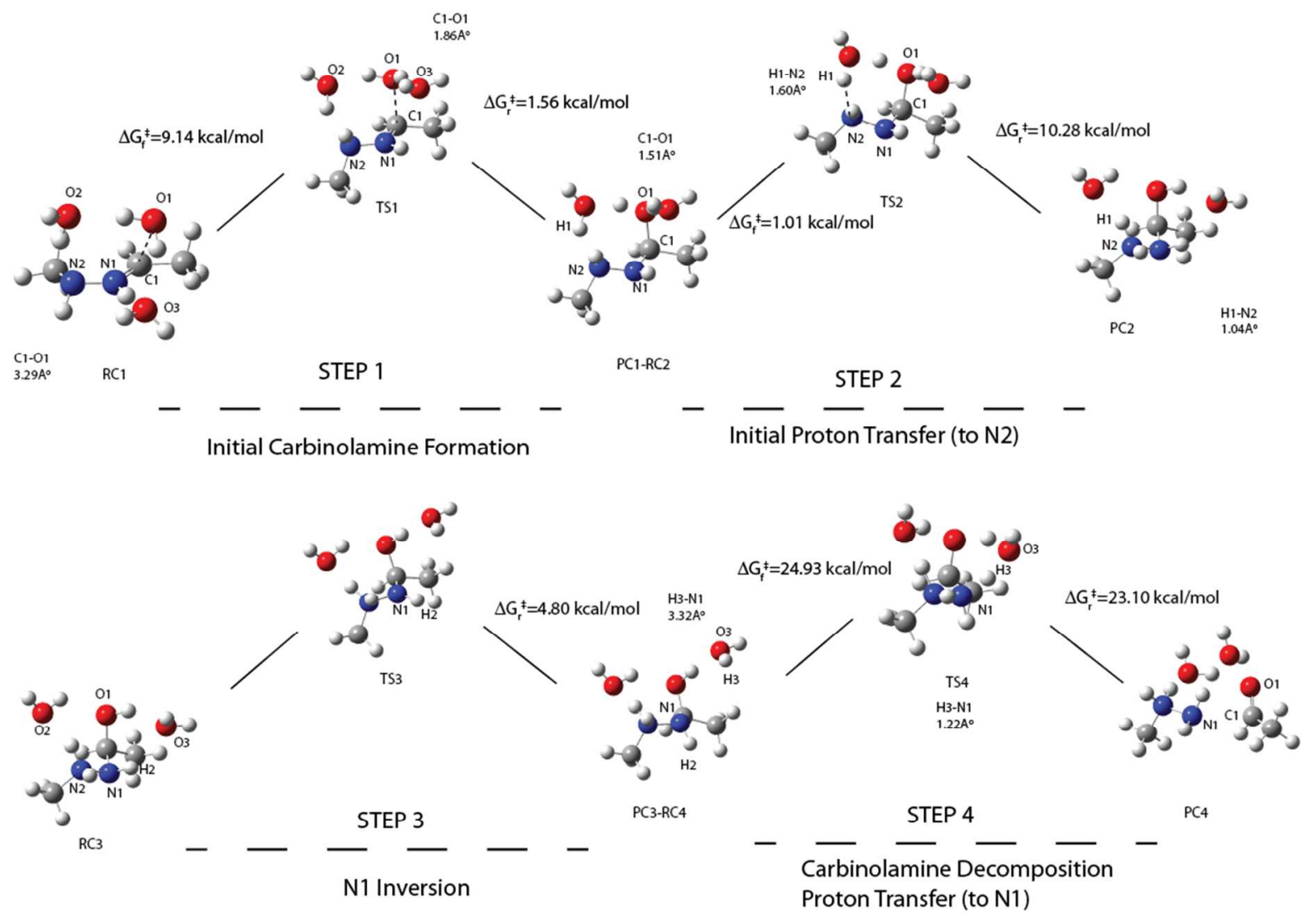

Figure S6. Four-step hydrazone hydrolysis process based on the reaction of three $\mathrm{H}_{2} \mathrm{O}$ molecules with the model compound 8 through Pathway 2. In Step 1, C1-O1 bond formation lead to the formation of initial carbinolamine intermediate termed as RC1-PC1 (product complex1-reactant complex2). An internal proton (H1) transfer (Step 2) occurs to N2 position which is followed by the inversion of N1 (Step 3). The resultant carbinolamine decomposes into corresponding products through a second proton (H2) transfer process to N1 (Step 4). 


\section{Supporting Tables}

Table S1. Energy Profile in Carbinolamine Formation Process for Pathway 1 and Pathway 2

\begin{tabular}{|c|c|c|c|c|c|c|c|}
\hline \multicolumn{8}{|c|}{ Carbinolamine Formation } \\
\hline \multirow[b]{2}{*}{$\begin{array}{l}\text { Reaction } \\
\text { Path }\end{array}$} & \multirow[b]{2}{*}{$\begin{array}{l}\text { \# of } \\
\mathrm{H}_{2} \mathrm{O}\end{array}$} & \multicolumn{2}{|c|}{ Step 1} & \multicolumn{2}{|c|}{ Step 2} & \multicolumn{2}{|c|}{ Step 3} \\
\hline & & $\Delta \mathrm{G}_{\mathrm{f}}{ }^{\dagger}$ & $\Delta \mathrm{G}_{\mathrm{r}}{ }^{+}$ & $\Delta \mathrm{G}_{\mathrm{f}}{ }^{\dagger}$ & $\Delta \mathrm{G}_{\mathrm{r}}{ }^{*}$ & $\Delta \mathrm{G}_{\mathrm{f}}{ }^{t}$ & $\Delta \mathrm{G}_{\mathrm{r}}{ }^{\dagger}$ \\
\hline \multirow{3}{*}{ Path 1} & $1 \mathrm{H}_{2} \mathrm{O}$ & 34.56 & 32.15 & \multicolumn{4}{|c|}{1 step-reaction } \\
\hline & $2 \mathrm{H}_{2} \mathrm{O}$ & 14.37 & 0.01 & 0.60 & 0.29 & 1.70 & 14.37 \\
\hline & $3 \mathrm{H}_{2} \mathrm{O}$ & 8.69 & 1.05 & 1.01 & 1.60 & 4.57 & 13.50 \\
\hline \multirow{3}{*}{ Path 2} & $1 \mathrm{H}_{2} \mathrm{O}$ & 19.68 & 21.45 & & 1 step & ction & \\
\hline & $2 \mathrm{H}_{2} \mathrm{O}$ & 10.65 & 0.75 & 0.05 & 12.47 & \multirow{2}{*}{\multicolumn{2}{|c|}{2 step-reaction }} \\
\hline & $3 \mathrm{H}_{2} \mathrm{O}$ & 9.14 & 1.56 & 1.01 & 10.28 & & \\
\hline
\end{tabular}

$\Delta \mathrm{G}_{\mathrm{f}}{ }^{\dagger}$ :Forward reaction Gibbs free energy of activation in $\mathrm{kcal} / \mathrm{mol}$

$\Delta \mathrm{G}_{\mathrm{r}}{ }^{\dagger}$ :Reverse reaction Gibbs free energy of activation in $\mathrm{kcal} / \mathrm{mol}$

Table S2. Energy Profile in Carbinolamine Decomposition Process for Pathway 1 and Pathway 2

\begin{tabular}{|c|c|c|c|c|c|}
\hline \multicolumn{6}{|c|}{ Carbinolamine Decomposition } \\
\hline \multirow[b]{2}{*}{$\begin{array}{l}\text { Reaction } \\
\text { Path }\end{array}$} & \multirow[b]{2}{*}{$\begin{array}{l}\# \text { of } \\
\mathrm{H}_{2} \mathrm{O}\end{array}$} & \multicolumn{2}{|c|}{ Step 1} & \multicolumn{2}{|c|}{ Step 2} \\
\hline & & $\Delta \mathrm{G}_{\mathrm{f}}{ }^{\ddagger}$ & $\Delta \mathrm{G}_{\mathrm{r}}^{*}$ & $\Delta \mathrm{G}_{\mathrm{f}}^{*}$ & $\Delta \mathrm{G}_{\mathrm{r}}{ }^{\ddagger}$ \\
\hline \multirow{3}{*}{ Path 1} & $1 \mathrm{H}_{2} \mathrm{O}$ & 14.84 & 16.73 & \multirow{3}{*}{\multicolumn{2}{|c|}{1 step-reaction }} \\
\hline & $2 \mathrm{H}_{2} \mathrm{O}$ & 16.30 & 15.68 & & \\
\hline & $3 \mathrm{H}_{2} \mathrm{O}$ & 19.17 & 16.23 & & \\
\hline \multirow{3}{*}{ Path 2} & $1 \mathrm{H}_{2} \mathrm{O}$ & 6.09 & 5.33 & 43.02 & 41.83 \\
\hline & $2 \mathrm{H}_{2} \mathrm{O}$ & 3.80 & 4.15 & 42.53 & 38.83 \\
\hline & $3 \mathrm{H}_{2} \mathrm{O}$ & 4.83 & 4.80 & 24.93 & 23.10 \\
\hline
\end{tabular}

$\Delta \mathrm{G}_{\mathrm{f}}{ }^{\dagger}$ :Forward reaction Gibbs free energy of activation in $\mathrm{kcal} / \mathrm{mol}$

$\Delta \mathrm{G}_{\mathrm{r}}{ }^{\dagger}$ :Reverse reaction Gibbs free energy of activation in $\mathrm{kcal} / \mathrm{mol}$ 
Table S3. Absolute Energies of Species and Imaginary Frequencies for Transition States for Path1-One $\mathrm{H}_{2} \mathrm{O}$ Molecule

(ZPE: Zero-point energy correction, H: Enthalpy, G: Gibbs free energy)

\begin{tabular}{lllll}
\hline Species & E+ZPE (au) & H (au) & G (au) & $\begin{array}{l}\text { Imaginary } \\
\text { Frequency }(\boldsymbol{i})\end{array}$ \\
\hline RC1 & -305.270836 & -305.259777 & -305.305528 & \\
\hline TS1 & -305.219502 & -305.21134 & -305.250459 & -1525.34 \\
\hline PC1-RC2 & -305.270646 & -305.262205 & -305.301693 & \\
\hline TS2 & -305.246234 & -305.237923 & -305.278038 & -842.32 \\
\hline PC2 & -305.268659 & -305.258075 & -305.304694 & \\
\hline
\end{tabular}

Table S4. Absolute Energies of Species and Imaginary Frequencies for Transition States for Path1-Two $\mathrm{H}_{2} \mathrm{O}$ Molecules (ZPE: Zero-point energy correction, H: Enthalpy, G: Gibbs free energy)

\begin{tabular}{lllll}
\hline Species & E+ZPE (au) & H (au) & G (au) & $\begin{array}{l}\text { Imaginary } \\
\text { Frequency }(\boldsymbol{i})\end{array}$ \\
\hline RC1 & -381.686301 & -381.672135 & -381.726522 & \\
\hline TS1 & -381.668116 & -381.656584 & -381.703614 & -154.15 \\
\hline PC1-RC2 & -381.667672 & -381.656045 & -381.703592 & \\
\hline TS2 & -381.666679 & -381.655067 & -381.702641 & -220.41 \\
\hline PC2-RC3 & -381.66739 & -381.656305 & -381.702179 & \\
\hline TS3 & -381.666086 & -381.656138 & -381.699428 & -178.90 \\
\hline PC3-RC4 & -381.689736 & -381.678169 & -381.72499 & \\
\hline TS4 & -381.664941 & -381.653501 & -381.700498 & -729.57 \\
\hline PC4 & -381.684679 & -381.670646 & -381.725482 & \\
\hline
\end{tabular}

Table S5. Absolute Energies of Species and Imaginary Frequencies for Transition States for Path1-Three $\mathrm{H}_{2} \mathrm{O}$ Molecules (ZPE: Zero-point energy correction, H: Enthalpy, G: Gibbs free energy)

\begin{tabular}{lllll}
\hline Species & E+ZPE (au) & H (au) & G (au) & $\begin{array}{l}\text { Imaginary } \\
\text { Frequency }(\boldsymbol{i})\end{array}$ \\
\hline RC1 & -458.102691 & -458.086518 & -458.143951 & \\
\hline
\end{tabular}




\begin{tabular}{lllll}
\hline TS1 & -458.090546 & -458.076 & -458.130104 & -215.38 \\
\hline PC1-RC2 & -458.093488 & -458.080 & -458.131776 & \\
\hline TS2 & -458.09208 & -458.078344 & -458.13015 & -271.43 \\
\hline PC2-RC3 & -458.095116 & -458.081686 & -458.1327 & \\
\hline TS3 & -458.089207 & -458.076911 & -458.125409 & -355.31 \\
\hline PC3-RC4 & -458.10718 & -458.092566 & -458.146926 & \\
\hline TS4 & -458.078612 & -458.064982 & -458.116678 & -626.02 \\
\hline PC4 & -458.09996 & -458.083642 & -458.142546 & \\
\hline
\end{tabular}

Table S6. Absolute Energies of Species and Imaginary Frequencies for Transition States for Path2-One $\mathrm{H}_{2} \mathrm{O}$ Molecule (ZPE: Zero-point energy correction, H: Enthalpy, G: Gibbs free energy)

\begin{tabular}{lllll}
\hline Species & E+ZPE (au) & H (au) & G (au) & $\begin{array}{l}\text { Imaginary } \\
\text { Frequency }(\boldsymbol{i})\end{array}$ \\
\hline RC1 & -305.269067 & -305.257834 & -305.304271 & \\
\hline TS1 & -305.242012 & -305.234 & -305.272905 & -139.43 \\
\hline PC1-RC2 & -305.275501 & -305.267 & -305.307083 & \\
\hline TS2 & -305.266286 & -305.257901 & -305.297511 & -643.29 \\
\hline PC2-RC3 & -305.274518 & -305.265984 & -305.30601 & \\
\hline TS3 & -305.198201 & -305.189967 & -305.229305 & -1448.71 \\
\hline PC3 & -305.259555 & -305.248767 & -305.295967 & \\
\hline
\end{tabular}

Table S7. Absolute Energies of Species and Imaginary Frequencies for Transition States for Path2-Two $\mathrm{H}_{2} \mathrm{O}$ Molecules (ZPE: Zero-point energy correction, H: Enthalpy, G: Gibbs free energy)

\begin{tabular}{lllll}
\hline Species & E+ZPE (au) & H (au) & G (au) & $\begin{array}{l}\text { Imaginary } \\
\text { Frequency }(\boldsymbol{i})\end{array}$ \\
\hline RC1 & -381.684304 & -381.670824 & -381.722269 & \\
\hline TS1 & -381.671105 & -381.660 & -381.705293 & -199.51 \\
\hline PC1-RC2 & -381.672119 & -381.661 & -381.706494 & \\
\hline TS2 & -381.673104 & -381.662907 & -381.706546 & -111.30 \\
\hline PC2-RC3 & -381.692566 & -381.681857 & -381.726418 & \\
\hline TS3 & -381.686836 & -381.67562 & -381.721651 & -553.59 \\
\hline PC3-RC4 & -381.693097 & -381.681622 & -381.728272 & \\
\hline TS4 & -381.626553 & -381.616008 & -381.660503 & -1475.05 \\
\hline PC4 & -381.68402 & -381.67092 & -381.722389 & \\
\hline
\end{tabular}


Table S8. Absolute Energies of Species and Imaginary Frequencies for Transition States for Path2-Three $\mathrm{H}_{2} \mathrm{O}$ Molecules (ZPE: Zero-point energy correction, H: Enthalpy, G: Gibbs free energy)

\begin{tabular}{lllll}
\hline Species & E+ZPE (au) & H (au) & G (au) & $\begin{array}{l}\text { Imaginary } \\
\text { Frequency }(\boldsymbol{i})\end{array}$ \\
\hline RC1 & -458.102691 & -458.086518 & -458.143951 & \\
\hline TS1 & -458.090546 & -458.076 & -458.130104 & -215.38 \\
\hline PC1-RC2 & -458.093488 & -458.080 & -458.131776 & \\
\hline TS2 & -458.094777 & -458.081545 & -458.132525 & -673.77 \\
\hline PC2-RC3 & -458.109722 & -458.09497 & -458.149409 & \\
\hline TS3 & -458.101493 & -458.086895 & -458.141713 & -574.14 \\
\hline PC3-RC4 & -458.108618 & -458.093639 & -458.149358 & \\
\hline TS4 & -458.07107 & -458.059083 & -458.106853 & -951.84 \\
\hline PC4 & -458.09958 & -458.083028 & -458.143658 & \\
\hline
\end{tabular}

Cartesian Coordinates Corresponding to the Optimized Geometries of All Species involved in Both Pathways

Pathway 1 - One $\mathrm{H}_{2} \mathrm{O}$ Molecule as Reactant

$\underline{\text { RC1 (Reactant Complex for Step 1) }}$

$\begin{array}{llll}\mathrm{C} & 1.00943200 & 0.54816300 & -0.43561300 \\ \mathrm{H} & 1.02401900 & 0.03473100 & -1.39145100 \\ \mathrm{~N} & -0.12711400 & 0.60651800 & 0.14376900 \\ \mathrm{~N} & -1.25173900 & -0.03252800 & -0.38042200 \\ \mathrm{H} & -1.14452000 & -1.01691900 & -0.13258000 \\ \mathrm{H} & -0.21886500 & 1.07504800 & 1.04914200 \\ \mathrm{O} & 0.57388400 & -2.21466700 & 0.11248800\end{array}$




$\begin{array}{llll}\mathrm{H} & 0.67670600 & -2.99491700 & -0.44086200 \\ \mathrm{H} & 0.89770000 & -2.47920800 & 0.97921300 \\ \mathrm{C} & -2.48663700 & 0.55346500 & 0.13880800 \\ \mathrm{H} & -3.31010300 & -0.04755500 & -0.24122100 \\ \mathrm{H} & -2.52782200 & 0.55979200 & 1.23333400 \\ \mathrm{H} & -2.59152800 & 1.56953500 & -0.23966100 \\ \mathrm{C} & 2.23798000 & 1.11295400 & 0.14937200 \\ \mathrm{H} & 2.93892500 & 0.29092600 & 0.31960600 \\ \mathrm{H} & 2.70002700 & 1.78266200 & -0.57852300 \\ \mathrm{H} & 2.05170500 & 1.63782800 & 1.08426700\end{array}$

\section{TS1 (Transition State for Step 1)}

$\begin{array}{llll}\mathrm{C} & 0.82119700 & 0.12639500 & -0.33712500 \\ \mathrm{H} & 0.45428300 & 0.14364800 & -1.35990100 \\ \mathrm{~N} & -0.24159200 & -0.25859400 & 0.60182600 \\ \mathrm{~N} & -1.45657800 & -0.62957200 & -0.03217000 \\ \mathrm{H} & -1.89832600 & -1.31312400 & 0.57362000 \\ \mathrm{H} & -0.36198600 & 0.40320100 & 1.37369600 \\ \mathrm{O} & 1.60016700 & -1.12778100 & -0.11459700 \\ \mathrm{H} & 1.63456200 & -1.71701400 & -0.88652400 \\ \mathrm{H} & 0.69620200 & -1.28540200 & 0.62208600 \\ \mathrm{C} & -2.33542400 & 0.52266700 & -0.23849900 \\ \mathrm{H} & -3.27130500 & 0.15774100 & -0.65942400 \\ \mathrm{H} & -2.54412200 & 1.06576200 & 0.69029500 \\ \mathrm{H} & -1.87559600 & 1.20319100 & -0.95697300 \\ \mathrm{C} & 1.63813700 & 1.31164500 & 0.07759700 \\ \mathrm{H} & 2.50176400 & 1.40544400 & -0.57903400\end{array}$



$\mathrm{H}$
$-0.00814300$
$\mathrm{H}$
1.10765100

Structure of TS1 with Displacement Vectors with Full Scale Corresponding to Imaginary Frequency

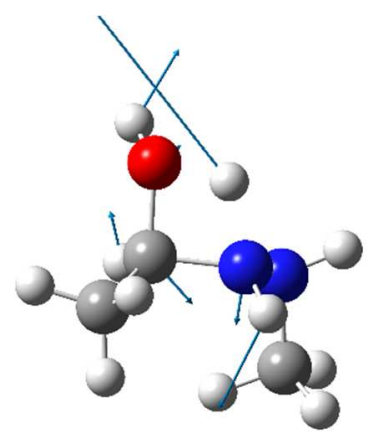

$\underline{\text { PC1-RC2 (Product Complex for Step 1-Reactant Complex for Step 2) }}$

$\begin{array}{llll}\mathrm{C} & 0.86850000 & -0.02815700 & -0.34155000 \\ \mathrm{H} & 0.43378800 & 0.11650700 & -1.33268800 \\ \mathrm{~N} & -0.21879300 & 0.43253500 & 0.63268400 \\ \mathrm{~N} & -1.41703000 & -0.33784900 & 0.43988300 \\ \mathrm{H} & -1.88666900 & -0.37462400 & 1.33951500 \\ \mathrm{H} & -0.38328700 & 1.44534600 & 0.54838200 \\ \mathrm{O} & 1.12506100 & -1.35082400 & -0.05586000 \\ \mathrm{H} & 0.34063600 & -1.87683600 & -0.26120900 \\ \mathrm{H} & 0.13522400 & 0.25673500 & 1.57937800 \\ \mathrm{C} & -2.29023900 & 0.28272500 & -0.56299900 \\ \mathrm{H} & -3.20432100 & -0.30710800 & -0.58569800\end{array}$




$\begin{array}{llll}\mathrm{H} & -2.53721200 & 1.32178300 & -0.32341000 \\ \mathrm{H} & -1.82568700 & 0.22865300 & -1.54639400 \\ \mathrm{C} & 2.10752800 & 0.80246800 & -0.13064400 \\ \mathrm{H} & 2.86025600 & 0.47368800 & -0.84591600 \\ \mathrm{H} & 1.90660000 & 1.86032300 & -0.30071200 \\ \mathrm{H} & 2.49620400 & 0.65710800 & 0.87881600\end{array}$

\section{TS2 (Transition State for Step 1)}

$\begin{array}{lrrr}\mathrm{C} & -1.15783500 & 0.29475000 & 0.46081700 \\ \mathrm{~N} & 0.26389200 & -0.95790800 & 0.12858400 \\ \mathrm{~N} & 1.18498000 & -0.10526400 & -0.52574000 \\ \mathrm{H} & -0.03837700 & -1.71675500 & -0.48085200 \\ \mathrm{H} & 1.37935200 & -0.44513500 & -1.46675700 \\ \mathrm{H} & -1.09255000 & 0.24957200 & 1.55307100 \\ \mathrm{O} & -0.66047400 & 1.32596200 & -0.12045700 \\ \mathrm{H} & 0.45679800 & 0.86415100 & -0.53290400 \\ \mathrm{H} & 0.65943700 & -1.35298600 & 0.98149000 \\ \mathrm{C} & -2.34251100 & -0.39893400 & -0.14576400 \\ \mathrm{H} & -2.50114400 & -1.38183200 & 0.29916900 \\ \mathrm{H} & -3.22186700 & 0.21596400 & 0.06245000 \\ \mathrm{H} & -2.22525200 & -0.48062200 & -1.22733200 \\ \mathrm{C} & 2.42879400 & 0.14319700 & 0.23787000 \\ \mathrm{H} & 3.02063500 & 0.85422800 & -0.33322500 \\ \mathrm{H} & 2.15753300 & 0.58094200 & 1.19739200 \\ \mathrm{H} & 2.97643700 & -0.78710300 & 0.37370900\end{array}$

Structure of TS2 with the Displacement Vectors with Full Scale Corresponding to Imaginary Frequency 


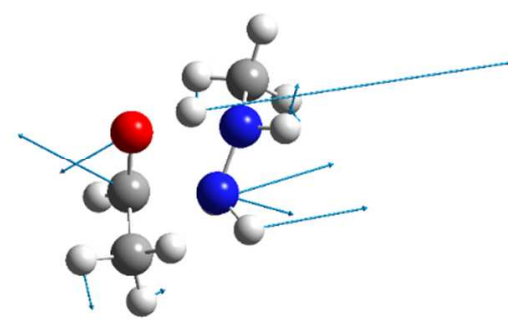

\section{PC2 (Product Complex for Step 2)}

$\mathrm{C}$

$\mathrm{N}$

$\begin{array}{lll}1.96704900 & 0.19368400 & 0.28635200\end{array}$

$-2.31558800-1.07866200 \quad-0.26355800$

$\mathrm{N}$

$\begin{array}{lll}-1.65632600 & -0.00432300 & 0.42222700\end{array}$

$\mathrm{H} \quad \begin{array}{llll}\mathrm{H} & -2.09268700 & -1.93714700 & 0.23511300\end{array}$

$\begin{array}{llll}\mathrm{H} & -1.93563800 & 0.07004700 & 1.40509000\end{array}$

$\mathrm{H}$

$\begin{array}{lll}1.82857700 & 0.99407500 & 1.03119600\end{array}$

$\mathrm{O}$

$1.00409300 \quad-0.43837700 \quad-0.09987300$

$\mathrm{H}$

$\begin{array}{lll}-0.63091000 & -0.20078700 & 0.38359100\end{array}$

$\mathrm{H}$

$-3.31747500 \quad-0.92633000 \quad-0.16886500$

$\mathrm{C}$

$3.35624500 \quad-0.04669800-0.18645100$

$\mathrm{H}$

$3.75225600 \quad 0.88304600 \quad-0.60373600$

$\mathrm{H}$

$\begin{array}{lll}3.98051600 & -0.29485200 & 0.67652900\end{array}$

$\mathrm{H}$

$\begin{array}{lll}3.38868200 & -0.84504000 & -0.92478700\end{array}$

C

$\begin{array}{llll}-1.88808700 & 1.28673700 & -0.27415200\end{array}$

$\mathrm{H}$

$\begin{array}{lll}-1.33229700 & 2.05793600 & 0.25402800\end{array}$

$\mathrm{H}$

$\begin{array}{llll}-1.52906900 & 1.17647400 & -1.29380400\end{array}$

$\mathrm{H}$

$\begin{array}{lll}-2.95253900 & 1.50814200 & -0.26054400\end{array}$ 


\section{Pathway 1 - Two $\mathrm{H}_{2} \mathrm{O}$ Molecule as Reactant \\ $\underline{\text { RC1 (Reactant Complex for Step 1) }}$}

C

$\begin{array}{llll}-0.13458200 & -1.53671700 & -0.09025400\end{array}$

$\mathrm{N}$

$\begin{array}{llll}0.49575000 & -0.43133100 & -0.00894000\end{array}$

$\mathrm{N}$

$\begin{array}{lll}1.69642300 & -0.33247200 & 0.71661000\end{array}$

$\mathrm{H} \quad-0.25729900 \quad 2.85001100 \quad-0.60611600$

$\mathrm{H}$

$\mathrm{H}$

$\mathrm{O}$

$\mathrm{H}$

$\mathrm{H}$

$\mathrm{O}$

$\mathrm{H}$

$\mathrm{H}$

C

$\mathrm{H}$

$\mathrm{H}$

$\mathrm{H}$

C

$\mathrm{H}$

$\mathrm{H}$

$\mathrm{H}$

$$
\begin{array}{lll}
1.47726500 & 0.21255700 & 1.54840600
\end{array}
$$

$\begin{array}{lll}0.32640900 & -2.38243400 & 0.41242800\end{array}$

$\begin{array}{lll}-2.02396900 & 0.56722800 & 1.22818300\end{array}$

$\begin{array}{lll}-2.89007500 & 0.76001500 & 1.59636700\end{array}$

$\begin{array}{llll}0.11463700 & 0.42862800 & -0.44362400\end{array}$

$\begin{array}{llll}-0.71500400 & 2.02927300 & -0.82048200\end{array}$

$\begin{array}{lll}-1.83764200 & 1.28916600 & 0.61254500\end{array}$

$\begin{array}{llll}-1.09461400 & 2.16459100 & -1.69607300\end{array}$

$\begin{array}{lll}-1.41320400 & -1.67516300 & -0.80765500\end{array}$

$\begin{array}{lll}-1.32543700 & -2.49331700 & -1.52570300\end{array}$

$\begin{array}{lll}-2.17582500 & -1.95608700 & -0.07668700\end{array}$

$\begin{array}{llll}-1.71147200 & -0.75454500 & -1.30416900\end{array}$

$\begin{array}{llll}2.71326900 & 0.36063900 & -0.08591900\end{array}$

$\begin{array}{lll}3.58608200 & 0.49637600 & 0.55002900\end{array}$

$2.98540800 \quad-0.26989800 \quad-0.93120900$

$\begin{array}{llll}2.37623800 & 1.33699800 & -0.44853300\end{array}$

\section{TS1 (Transition State for Step 1)}

C $\quad 0.08379700 \quad 0.91471300 \quad 0.32624400$

$\mathrm{N} \quad \begin{array}{llll}0.78958600 & 0.17624800 & -0.39854100\end{array}$

N $\quad \begin{array}{llll}-1.73317600 & -0.58981400 & 0.30556000\end{array}$

$\mathrm{H} \quad 2.24104100 \quad-1.77555500 \quad-1.23434700$ 


$\begin{array}{llll}\mathrm{H} & -1.45810300 & -1.56751500 & 0.26376800 \\ \mathrm{H} & -0.37570400 & 1.35274700 & 1.20697200 \\ \mathrm{O} & 1.16958600 & -0.06083700 & 1.15582500 \\ \mathrm{H} & 0.69113000 & -0.65906500 & 1.74895500 \\ \mathrm{H} & -0.50459300 & -0.15062000 & -1.31716500 \\ \mathrm{O} & 2.63152400 & -1.36822200 & -0.45314500 \\ \mathrm{H} & 1.74503400 & -0.61062500 & 0.51610500 \\ \mathrm{H} & 3.46041100 & -0.96570200 & -0.73517100 \\ \mathrm{C} & 1.00186500 & 1.80567600 & -0.45849300 \\ \mathrm{H} & 0.40709500 & 2.60391700 & -0.90268600 \\ \mathrm{H} & 1.74986200 & 2.24371500 & 0.19872000 \\ \mathrm{H} & 1.49980800 & 1.25116300 & -1.25599700 \\ \mathrm{C} & -3.07537300 & -0.42723600 & -0.25446800 \\ \mathrm{H} & -3.75033500 & -1.08612000 & 0.29181900 \\ \mathrm{H} & -3.39509600 & 0.60444500 & -0.10986900 \\ \mathrm{H} & -3.12182000 & -0.67225900 & -1.32137100\end{array}$

Structure of TS1 with the Displacement Vectors Corresponding to Imaginary Frequency

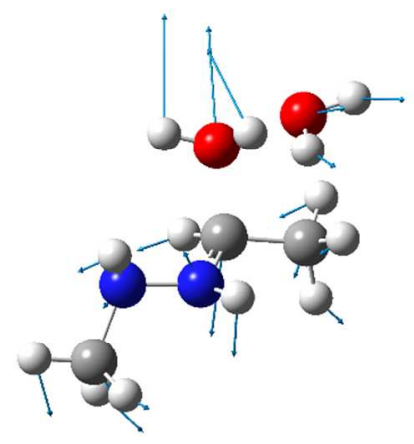

PC1-RC2 (Product Complex for Step 1-Reactant Complex for Step 2)

$\begin{array}{llll}\text { C } & 0.11308000 & 0.87639800 & 0.35496600\end{array}$ 


\begin{tabular}{|c|c|c|c|}
\hline $\mathrm{N}$ & -0.81388700 & 0.37251800 & -0.58291700 \\
\hline $\mathrm{N}$ & -1.58845400 & -0.68175100 & -0.01014200 \\
\hline $\mathrm{H}$ & 2.45552000 & -1.55671800 & -1.23203900 \\
\hline $\mathrm{H}$ & -1.41319200 & -1.53265000 & -0.53573000 \\
\hline $\mathrm{H}$ & -0.41300700 & 1.31171900 & 1.20273700 \\
\hline $\mathrm{O}$ & 0.84960400 & -0.27055400 & 1.02657200 \\
\hline $\mathrm{H}$ & 0.15086600 & -0.92528200 & 1.23742800 \\
\hline $\mathrm{H}$ & -0.38907900 & 0.11047000 & -1.46755600 \\
\hline $\mathrm{O}$ & 2.63490500 & -1.31246400 & -0.31612500 \\
\hline $\mathrm{H}$ & 1.60206700 & -0.71235300 & 0.42743400 \\
\hline $\mathrm{H}$ & 3.50638800 & -0.89957600 & -0.29372400 \\
\hline $\mathrm{C}$ & 1.14181700 & 1.79732100 & -0.24152200 \\
\hline $\mathrm{H}$ & 0.63885600 & 2.69489800 & -0.59955900 \\
\hline $\mathrm{H}$ & 1.87400000 & 2.08141800 & 0.51299700 \\
\hline $\mathrm{H}$ & 1.65283400 & 1.32182000 & -1.08126400 \\
\hline $\mathrm{C}$ & -3.01856300 & -0.37025400 & -0.04548600 \\
\hline $\mathrm{H}$ & -3.56144500 & -1.22012300 & 0.36928300 \\
\hline $\mathrm{H}$ & -3.20563800 & 0.50264700 & 0.57980800 \\
\hline $\mathrm{H}$ & -3.37585700 & -0.16828600 & -1.05973300 \\
\hline
\end{tabular}

\section{$\underline{\text { TS2 (Transition State for Step 2) }}$}

$\begin{array}{llll}\mathrm{C} & 0.07383800 & 0.79535000 & 0.34656500 \\ \mathrm{~N} & -0.64818100 & -0.12055200 & -0.37697600 \\ \mathrm{~N} & -1.71857000 & -0.76021000 & 0.25920900 \\ \mathrm{H} & 2.20434500 & -1.61566300 & -1.23207400 \\ \mathrm{H} & -1.63873600 & -1.75695200 & 0.09069700 \\ \mathrm{H} & -0.47840500 & 1.20732300 & 1.18942600 \\ \mathrm{O} & 1.22487400 & 0.04357500 & 1.15543100\end{array}$




$\begin{array}{llll}\mathrm{H} & 0.82019100 & -0.57073000 & 1.78806500 \\ \mathrm{H} & -0.64352800 & -0.06026000 & -1.38909800 \\ \mathrm{O} & 2.63542400 & -1.23790500 & -0.45665100 \\ \mathrm{H} & 1.81473900 & -0.50105300 & 0.49514100 \\ \mathrm{H} & 3.46086700 & -0.84061300 & -0.75724000 \\ \mathrm{C} & 0.81503300 & 1.81311200 & -0.47733300 \\ \mathrm{H} & 0.08764200 & 2.49444800 & -0.91916900 \\ \mathrm{H} & 1.49843900 & 2.38229100 & 0.14944300 \\ \mathrm{H} & 1.37716600 & 1.32936000 & -1.27861300 \\ \mathrm{C} & -3.01591700 & -0.26486800 & -0.20119400 \\ \mathrm{H} & -3.79900200 & -0.85008400 & 0.28128200 \\ \mathrm{H} & -3.12390800 & 0.77892500 & 0.09704000 \\ \mathrm{H} & -3.13264900 & -0.33858600 & -1.28900600\end{array}$

Structure of TS2 with the Displacement Vectors Corresponding to Imaginary Frequency

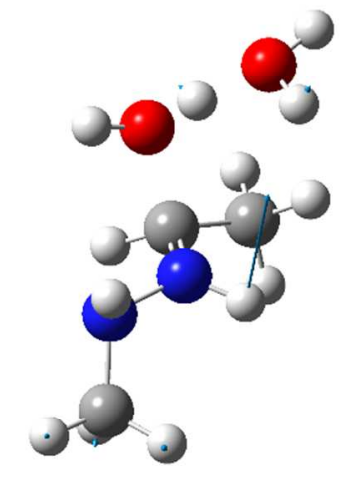

PC2-RC3 (Product Complex for Step 2-Reactant Complex for Step 3)

C

$0.16936500 \quad 0.87543300 \quad 0.27478400$

$\mathrm{N} \quad-0.47705300 \quad-0.01680600 \quad-0.64547700$

$\mathrm{N} \quad-1.47177400 \quad-0.76621500 \quad 0.03979200$ 


\begin{tabular}{llll}
$\mathrm{H}$ & 1.59690100 & -1.47711900 & -1.13400600 \\
$\mathrm{H}$ & -1.50243500 & -1.68392900 & -0.38917100 \\
$\mathrm{H}$ & -0.51898700 & 1.39695500 & 0.94440500 \\
$\mathrm{O}$ & 0.93197200 & -0.00732500 & 1.18978100 \\
$\mathrm{H}$ & 0.27185100 & -0.56780500 & 1.64253400 \\
$\mathrm{H}$ & -0.84425700 & 0.48942600 & -1.45127000 \\
$\mathrm{O}$ & 2.11386500 & -1.50252700 & -0.31551400 \\
$\mathrm{H}$ & 1.54235100 & -0.67098300 & 0.57028100 \\
$\mathrm{H}$ & 3.04541800 & -1.38996500 & -0.54177900 \\
$\mathrm{C}$ & 1.13153400 & 1.81512500 & -0.39952900 \\
$\mathrm{H}$ & 0.56569900 & 2.51890300 & -1.01098700 \\
$\mathrm{H}$ & 1.68968700 & 2.37554000 & 0.34886700 \\
$\mathrm{H}$ & 1.82423000 & 1.26939400 & -1.04185500 \\
$\mathrm{C}$ & -2.79688300 & -0.14568500 & 0.00425900 \\
$\mathrm{H}$ & -3.50352500 & -0.81274200 & 0.49780100 \\
$\mathrm{H}$ & -2.77542700 & 0.79714100 & 0.55296900 \\
$\mathrm{H}$ & -3.14051000 & 0.04591000 & -1.01920800 \\
& & & \\
\hline & & & \\
$\mathrm{H}$ & & &
\end{tabular}

\section{TS3 (Transition State for Step 3)}

$\begin{array}{llll}\mathrm{C} & 0.30225300 & 0.86426500 & 0.27165300 \\ \mathrm{~N} & -0.40356100 & -0.06403800 & -0.60341300 \\ \mathrm{~N} & -1.46139500 & -0.69449500 & 0.11457200 \\ \mathrm{H} & 0.94178800 & -1.36533800 & -0.92022500 \\ \mathrm{H} & -1.57721700 & -1.62296700 & -0.27576000 \\ \mathrm{H} & -0.38782300 & 1.44372200 & 0.88868400 \\ \mathrm{O} & 1.09574900 & 0.06525400 & 1.18887000 \\ \mathrm{H} & 0.51364100 & -0.28778400 & 1.87800400 \\ \mathrm{H} & -0.74482900 & 0.42674500 & -1.43195800\end{array}$




$\begin{array}{llll}\mathrm{O} & 1.66574400 & -1.71238700 & -0.32873600 \\ \mathrm{H} & 1.59440800 & -0.95801300 & 0.46760100 \\ \mathrm{H} & 2.51797300 & -1.74922200 & -0.78803800 \\ \mathrm{C} & 1.23458400 & 1.75703900 & -0.50879500 \\ \mathrm{H} & 0.65466300 & 2.41089300 & -1.16132200 \\ \mathrm{H} & 1.81076900 & 2.37286500 & 0.17988600 \\ \mathrm{H} & 1.92118600 & 1.16925400 & -1.12166500 \\ \mathrm{C} & -2.72420000 & 0.04004300 & 0.02950700 \\ \mathrm{H} & -3.49128700 & -0.53451300 & 0.54813800 \\ \mathrm{H} & -2.62387800 & 1.00443000 & 0.52975700 \\ \mathrm{H} & -3.04247100 & 0.20864200 & -1.00647900\end{array}$

Structure of TS3 with the Displacement Vectors Corresponding to Imaginary Frequency

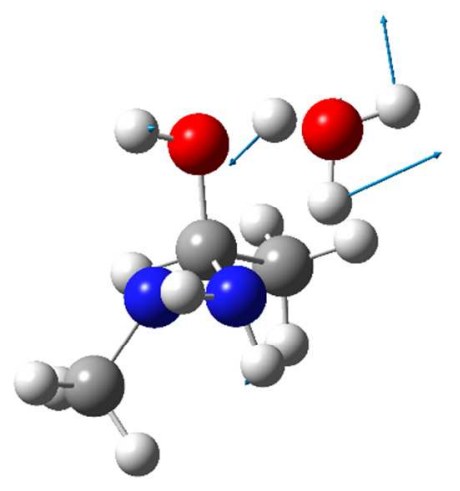

PC3-RC4 (Product Complex for Step 3-Reactant Complex for Step 4)

$\mathrm{C}$

$$
\begin{array}{lll}
0.34687500 & 1.02315700 & 0.17595600
\end{array}
$$

$\mathrm{N}$

$\begin{array}{lll}-0.34078900 & -0.09249700 & -0.59441700\end{array}$

$\mathrm{N}$

$\begin{array}{lll}-1.41713800 & -0.64405500 & 0.17644800\end{array}$

$\mathrm{H}$

$$
0.38254000-0.84175100-0.70320500
$$




$\begin{array}{llll}\mathrm{H} & -1.49639600 & -1.61954500 & -0.09681500 \\ \mathrm{H} & -0.42425500 & 1.76963400 & 0.37519900 \\ \mathrm{O} & 0.84990800 & 0.45965200 & 1.33638300 \\ \mathrm{H} & 0.11026400 & 0.16684400 & 1.88546800 \\ \mathrm{H} & -0.64212100 & 0.23099300 & -1.52252200 \\ \mathrm{O} & 1.53512700 & -1.98759400 & -0.16753400 \\ \mathrm{H} & 2.00772000 & -1.63424700 & 0.59526900 \\ \mathrm{H} & 2.17563400 & -2.48380600 & -0.68659600 \\ \mathrm{C} & 1.47369500 & 1.57293500 & -0.66125300 \\ \mathrm{H} & 1.10022900 & 1.96312300 & -1.60876300 \\ \mathrm{H} & 1.94837300 & 2.38559800 & -0.11315300 \\ \mathrm{H} & 2.21491300 & 0.79420300 & -0.85164600 \\ \mathrm{C} & -2.67711200 & 0.06215400 & -0.07384700 \\ \mathrm{H} & -3.45097100 & -0.46372500 & 0.48192200 \\ \mathrm{H} & -2.61504600 & 1.07999400 & 0.30939700 \\ \mathrm{H} & -2.94642200 & 0.08260900 & -1.13470700\end{array}$

\section{TS4 (Transition State for Step 4)}

$\begin{array}{lrrr}\mathrm{C} & -1.47913400 & -0.63694900 & -0.16225900 \\ \mathrm{~N} & 0.17126500 & -0.04711700 & -0.83019400 \\ \mathrm{~N} & 1.03339800 & -0.42950900 & 0.22503600 \\ \mathrm{H} & 0.20534700 & 0.97273700 & -0.92740700 \\ \mathrm{H} & 1.45589600 & 0.42512200 & 0.60421900 \\ \mathrm{H} & -1.67933900 & -1.37288800 & -0.94925300 \\ \mathrm{O} & -1.11718100 & -1.08747000 & 0.98805100 \\ \mathrm{H} & 0.17543800 & -0.84621800 & 0.93166300 \\ \mathrm{H} & 0.42495700 & -0.49739900 & -1.70888500 \\ \mathrm{O} & 1.11660200 & 2.36267400 & 0.25754900\end{array}$




$\begin{array}{llll}\mathrm{H} & 0.66199200 & 2.90652600 & 0.90896800 \\ \mathrm{H} & 1.81009500 & 2.92057900 & -0.10961300 \\ \mathrm{C} & -2.32065800 & 0.60852100 & -0.23317900 \\ \mathrm{H} & -2.36128900 & 1.00728100 & -1.24760600 \\ \mathrm{H} & -3.33517600 & 0.33988400 & 0.07058100 \\ \mathrm{H} & -1.94218100 & 1.36363300 & 0.45790100 \\ \mathrm{C} & 2.02893700 & -1.46306900 & -0.13054800 \\ \mathrm{H} & 2.57371400 & -1.71147200 & 0.77690200 \\ \mathrm{H} & 1.49785700 & -2.34232900 & -0.49129300 \\ \mathrm{H} & 2.70980800 & -1.08171700 & -0.88895400\end{array}$

\section{Structure of TS4 with the Displacement Vectors Corresponding to Imaginary Frequency}

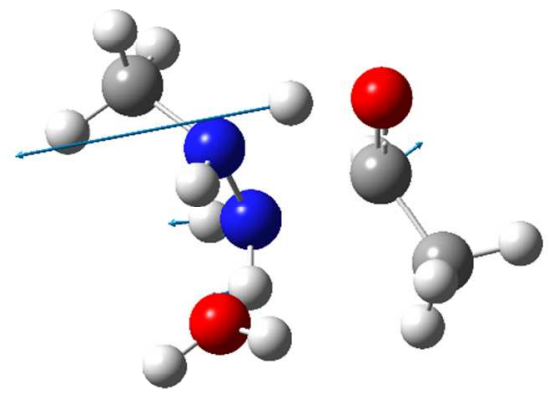

\section{PC4 (Product Complex for Step 4)}

$\begin{array}{lrrr}\mathrm{C} & -2.88048200 & 0.38469700 & -0.39564600 \\ \mathrm{~N} & 1.07883600 & -0.92056300 & 1.06022800\end{array}$




$\begin{array}{llll}\mathrm{N} & 0.90471400 & 0.11857400 & 0.08437100 \\ \mathrm{H} & 0.70535000 & -1.77459200 & 0.65189100 \\ \mathrm{H} & 1.49543000 & -0.06052700 & -0.73967700 \\ \mathrm{H} & -3.71125800 & 1.05386600 & -0.67237000 \\ \mathrm{O} & -1.74326100 & 0.79993800 & -0.46908600 \\ \mathrm{H} & -0.09458200 & 0.15520600 & -0.18564300 \\ \mathrm{H} & 2.08671100 & -1.05370600 & 1.13842600 \\ \mathrm{O} & 3.38497700 & -0.51792200 & -0.84640400 \\ \mathrm{H} & 3.68263500 & -1.34931500 & -1.22921700 \\ \mathrm{H} & 4.04841400 & 0.13141900 & -1.10005500 \\ \mathrm{C} & -3.25687900 & -0.98638900 & 0.04748600 \\ \mathrm{H} & -3.94294500 & -0.90509800 & 0.89479900 \\ \mathrm{H} & -3.81122800 & -1.47326700 & -0.75979200 \\ \mathrm{H} & -2.38367200 & -1.57684900 & 0.31828200 \\ \mathrm{C} & 1.27482400 & 1.43531400 & 0.65506300 \\ \mathrm{H} & 1.10870100 & 2.19301300 & -0.10759900 \\ \mathrm{H} & 0.64769200 & 1.61300800 & 1.52465800 \\ \mathrm{H} & 2.32539300 & 1.40291100 & 0.93660300 \\ & & & \\ \mathrm{H} & & & \end{array}$

\section{Pathway 1 - Three $\mathrm{H}_{2} \mathrm{O}$ Molecules as Reactant}

\section{$\underline{\text { RC1 (Reactant Complex for Step 1) }}$}

$\begin{array}{llll}\mathrm{C} & -0.36865600 & -1.44995000 & -0.51698500 \\ \mathrm{~N} & -0.44551500 & -0.58693600 & 0.42254200 \\ \mathrm{~N} & -1.52117900 & 0.24035800 & 0.70620500 \\ \mathrm{H} & 1.84383700 & 0.99174500 & 2.25205400 \\ \mathrm{H} & -1.58746300 & 0.31211400 & 1.71822100 \\ \mathrm{H} & -1.24883800 & -1.62971300 & -1.12388500 \\ \mathrm{O} & 1.98831700 & 0.75639100 & -1.16138900\end{array}$




\begin{tabular}{llll}
$\mathrm{H}$ & 1.26352600 & 1.39963100 & -1.09828500 \\
$\mathrm{H}$ & 0.39938700 & -0.37017500 & 0.98895000 \\
$\mathrm{O}$ & 1.89497700 & 0.18452400 & 1.72704400 \\
$\mathrm{H}$ & 2.27747800 & 0.63332500 & -0.24960300 \\
$\mathrm{H}$ & 2.42845700 & -0.43000500 & 2.24377500 \\
$\mathrm{C}$ & 0.88672600 & -2.18189700 & -0.79351700 \\
$\mathrm{H}$ & 0.67545800 & -3.25249900 & -0.81003400 \\
$\mathrm{H}$ & 1.24223500 & -1.89109100 & -1.78457500 \\
$\mathrm{H}$ & 1.65948100 & -1.96229800 & -0.05928200 \\
$\mathrm{C}$ & -2.78850800 & -0.15486300 & 0.09604500 \\
$\mathrm{H}$ & -3.55099900 & 0.49451400 & 0.51930500 \\
$\mathrm{H}$ & -2.74849000 & 0.01486300 & -0.98025400 \\
$\mathrm{H}$ & -3.04043000 & -1.19981300 & 0.30294700 \\
$\mathrm{O}$ & -0.27798200 & 2.40750700 & -0.80093200 \\
$\mathrm{H}$ & -0.22282700 & 3.31270500 & -0.48140700 \\
$\mathrm{H}$ & -0.84381900 & 1.93563200 & -0.17018800 \\
\hline & & & \\
\hline
\end{tabular}

\section{$\underline{\text { TS1 (Transition State for Step 1) }}$}

$\begin{array}{llll}\mathrm{C} & 0.33983000 & -0.28519800 & -0.99802000 \\ \mathrm{~N} & -0.32669500 & -0.87642100 & -0.01656000 \\ \mathrm{~N} & -1.51422700 & -0.31078200 & 0.48238000 \\ \mathrm{H} & 1.81447700 & -0.20184200 & 2.63276600 \\ \mathrm{H} & -1.37266100 & -0.14163700 & 1.47594400 \\ \mathrm{H} & -0.26500000 & 0.35305500 & -1.63342200 \\ \mathrm{O} & 1.27676700 & 1.16956000 & -0.32466900 \\ \mathrm{H} & 0.57336200 & 1.83036900 & -0.12333300 \\ \mathrm{H} & 0.14014800 & -1.58344800 & 0.54459800 \\ \mathrm{O} & 2.13186400 & -0.33359000 & 1.73296200 \\ \mathrm{H} & 1.65584900 & 0.83240400 & 0.51739100\end{array}$




$\begin{array}{llll}\mathrm{H} & 3.06071600 & -0.57280700 & 1.82011800 \\ \mathrm{C} & 1.47696000 & -1.03587600 & -1.60921900 \\ \mathrm{H} & 1.06279000 & -1.82993800 & -2.23346300 \\ \mathrm{H} & 2.07522400 & -0.37192600 & -2.22723100 \\ \mathrm{H} & 2.10293100 & -1.48308600 & -0.83565300 \\ \mathrm{C} & -2.65679200 & -1.21502600 & 0.29200800 \\ \mathrm{H} & -3.52705800 & -0.75153300 & 0.75537800 \\ \mathrm{H} & -2.83909900 & -1.32716000 & -0.77595300 \\ \mathrm{H} & -2.48730500 & -2.19844900 & 0.73959700 \\ \mathrm{O} & -1.04843700 & 2.43752800 & -0.20594400 \\ \mathrm{H} & -1.40888400 & 3.15467400 & 0.32328300 \\ \mathrm{H} & -1.54057500 & 1.63036600 & 0.03182600\end{array}$

Structure of TS1 with the Displacement Vectors Corresponding to Imaginary Frequency

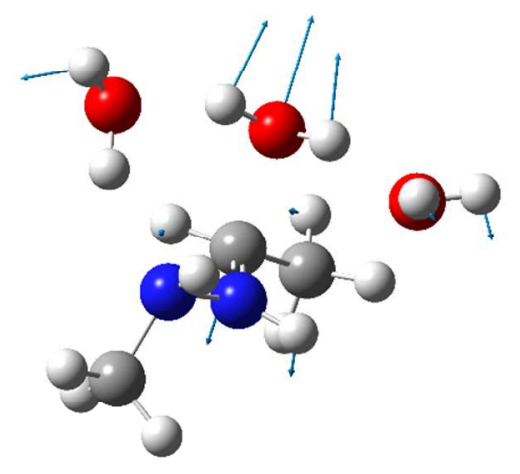




\section{PC1-RC2 (Product Complex for Step 1-Reactant Complex for Step 2)}

$\mathrm{C}$

$0.42528800-0.49304400-0.89495700$

$\mathrm{N} \quad \quad-0.41016100 \quad-0.99933300 \quad 0.12113900$

$\mathrm{N}$

$\begin{array}{lll}-1.49177900 & -0.15901600 & 0.50308400\end{array}$

$\mathrm{H}$

$\mathrm{H}$

$\mathrm{H}$

$\mathrm{O}$

$\mathrm{H}$

$\mathrm{H}$

$\mathrm{O}$

$\mathrm{H}$

$\mathrm{H}$

$\mathrm{C}$

$\mathrm{H}$

$\mathrm{H}$

$\mathrm{H}$

$\mathrm{C}$

$\mathrm{H}$

$\mathrm{H}$

$\mathrm{H}$

$\mathrm{O}$

$\mathrm{H}$

$\mathrm{H}$

$$
\begin{array}{lll}
1.85712700 & 0.72498100 & 2.51133300
\end{array}
$$

$\begin{array}{lll}-1.29407500 & 0.22794800 & 1.42368500\end{array}$

$\begin{array}{lll}-0.17277400 & -0.20866600 & -1.75996600\end{array}$

$\begin{array}{llll}1.08207700 & 0.81827000 & -0.52626200\end{array}$

$\begin{array}{llll}0.35982300 & 1.56805300 & -0.56346200\end{array}$

$\begin{array}{lll}0.09680500 & -1.38814700 & 0.91154400\end{array}$

$2.17793500 \quad 0.30898200 \quad 1.70304500$

$\begin{array}{llll}1.54256800 & 0.73454700 & 0.37691200\end{array}$

$\begin{array}{lll}3.14023500 & 0.30689500 & 1.76085100\end{array}$

$\begin{array}{llll}1.53985100 & -1.45122700 & -1.23512600\end{array}$

$\begin{array}{llll}1.11312800 & -2.37359800 & -1.62663500\end{array}$

$2.19350800 \quad-1.01064700 \quad-1.98567400$

$2.12639200 \quad-1.68421500 \quad-0.34319100$

$\begin{array}{lll}-2.74332500 & -0.92188400 & 0.56496500\end{array}$

$\begin{array}{lll}-3.53062200 & -0.25731700 & 0.92213700\end{array}$

$\begin{array}{llll}-2.99423000 & -1.26382100 & -0.43840400\end{array}$

$\begin{array}{lll}-2.66721400 & -1.78566500 & 1.23193400\end{array}$

$\begin{array}{llll}-0.91355800 & 2.25623700 & -0.76805400\end{array}$

$\begin{array}{llll}-1.08093500 & 3.08547200 & -0.30768700\end{array}$

$\begin{array}{lll}-1.47867500 & 1.56163600 & -0.36205300\end{array}$

\section{TS2 (Transition State for Step 2)}

$\mathrm{C}$

$\begin{array}{llll}0.26505200 & -0.59714500 & -0.77787300\end{array}$

$\mathrm{N}$

$\begin{array}{lll}-0.46515900 & -0.76229700 & 0.38755000\end{array}$ 


\begin{tabular}{|c|c|c|c|}
\hline $\mathrm{N}$ & -1.58972400 & 0.04016900 & 0.63049800 \\
\hline $\mathrm{H}$ & 3.47665400 & 0.57213100 & 1.43622300 \\
\hline $\mathrm{H}$ & -1.50532600 & 0.42646400 & 1.56691800 \\
\hline $\mathrm{H}$ & -0.36818200 & -0.31825200 & -1.62104900 \\
\hline $\mathrm{O}$ & 1.17984200 & 0.63997700 & -0.70119100 \\
\hline $\mathrm{H}$ & 0.55817100 & 1.45940100 & -0.69915500 \\
\hline $\mathrm{H}$ & -0.22271600 & -1.48731200 & 1.04875700 \\
\hline $\mathrm{O}$ & 2.55782700 & 0.28249500 & 1.41144600 \\
\hline $\mathrm{H}$ & 1.73686000 & 0.58701300 & 0.14704800 \\
\hline $\mathrm{H}$ & 2.17126100 & 0.53248200 & 2.25813600 \\
\hline $\mathrm{C}$ & 1.17593800 & -1.76246400 & -1.07294200 \\
\hline $\mathrm{H}$ & 0.57201000 & -2.64239300 & -1.29228000 \\
\hline $\mathrm{H}$ & 1.80338900 & -1.53507400 & -1.93252300 \\
\hline $\mathrm{H}$ & 1.81349300 & -1.97165100 & -0.21110300 \\
\hline $\mathrm{C}$ & -2.84661400 & -0.70846800 & 0.50999800 \\
\hline $\mathrm{H}$ & -3.67005100 & -0.04149200 & 0.76646600 \\
\hline $\mathrm{H}$ & -2.96225200 & -1.03413700 & -0.52377200 \\
\hline $\mathrm{H}$ & -2.86876200 & -1.58262900 & 1.16795800 \\
\hline $\mathrm{O}$ & -0.69862200 & 2.28579000 & -0.77947800 \\
\hline $\mathrm{H}$ & -0.72768500 & 3.14840300 & -0.35324400 \\
\hline $\mathrm{H}$ & -1.30131100 & 1.68430900 & -0.28602700 \\
\hline
\end{tabular}

Structure of TS2 with the Displacement Vectors Corresponding to Imaginary Frequency 


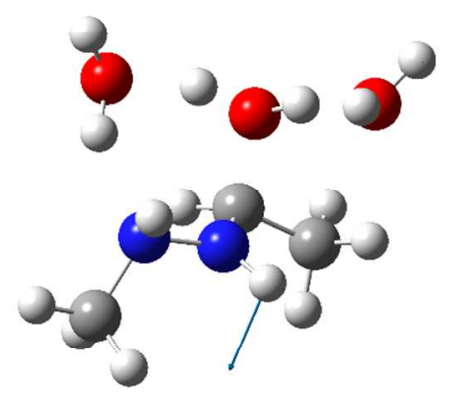

PC2-RC3 (Product Complex for Step 2-Reactant Complex for Step 3)

$\mathrm{C}$

$0.31607200-0.62923000-0.71521300$

$\mathrm{N} \quad-0.25810200 \quad-0.70443000 \quad 0.60139600$

$\mathrm{N} \quad-1.47736100 \quad 0.02484500 \quad 0.72224400$

$\begin{array}{llll}\mathrm{H} & 2.76207200 & 1.22618600 & 1.82254000\end{array}$

$\mathrm{H} \quad-1.54559300 \quad 0.31004800 \quad 1.69426500$

$\mathrm{H} \quad-0.43317500 \quad-0.52436300 \quad-1.50429800$

$\begin{array}{llll}\mathrm{O} & 1.08516300 & 0.62558500 & -0.80752100\end{array}$

H $\quad 0.39480300 \quad 1.42773600 \quad-0.84539900$

$\mathrm{H} \quad-0.37571400 \quad-1.67631800 \quad 0.88409400$

$\begin{array}{llll}\mathrm{O} & 2.33822400 & 0.49347800 & 1.36180600\end{array}$

$\begin{array}{llll}\mathrm{H} & 1.67363900 & 0.69156600 & 0.03593200\end{array}$

$\begin{array}{llll}\mathrm{H} & 1.62590700 & 0.16745100 & 1.92926700\end{array}$

C $\quad 1.25913800 \quad-1.77810800 \quad-0.97467700$

$\mathrm{H} \quad 0.69522500 \quad-2.71043100 \quad-1.01664400$

$\mathrm{H} \quad 1.76530800 \quad-1.63001000 \quad-1.92695400$

$\begin{array}{llll}\mathrm{H} & 2.00254400 & -1.84609300 & -0.17769600\end{array}$

$\begin{array}{llll}\text { C } & -2.65786700 & -0.76705300 & 0.35447700\end{array}$

H $\quad-3.54846200 \quad-0.16926100 \quad 0.54751600$ 


$\begin{array}{llll}\mathrm{H} & -2.62087300 & -1.00116100 & -0.71031500 \\ \mathrm{H} & -2.71776600 & -1.69932500 & 0.92595100 \\ \mathrm{O} & -0.79598400 & 2.19665500 & -0.83393900 \\ \mathrm{H} & -0.75402600 & 3.08813100 & -0.47121300 \\ \mathrm{H} & -1.29894500 & 1.62353900 & -0.20280400\end{array}$

\section{TS3 (Transition State for Step 3)}

$\begin{array}{llll}\mathrm{C} & 0.54450100 & -0.44394100 & -0.76085700 \\ \mathrm{~N} & -0.03375100 & -0.82505900 & 0.53956200 \\ \mathrm{~N} & -1.34471200 & -0.27917000 & 0.71460200 \\ \mathrm{H} & 1.03063900 & -0.07851000 & 1.59958100 \\ \mathrm{H} & -1.47962900 & -0.15489500 & 1.71323800 \\ \mathrm{H} & -0.23299800 & -0.40240500 & -1.52740700 \\ \mathrm{O} & 1.09021700 & 0.88350700 & -0.64418200 \\ \mathrm{H} & 0.35086500 & 1.53337200 & -0.78555600 \\ \mathrm{H} & -0.05141000 & -1.84300500 & 0.62352300 \\ \mathrm{O} & 1.65432200 & 0.73532900 & 1.68002400 \\ \mathrm{H} & 1.59571800 & 0.99053300 & 0.62022400 \\ \mathrm{H} & 2.53935000 & 0.48889200 & 1.98516700 \\ \mathrm{C} & 1.65434100 & -1.39487400 & -1.14148100 \\ \mathrm{H} & 1.25403800 & -2.39692100 & -1.29982600 \\ \mathrm{H} & 2.11843700 & -1.05185900 & -2.06469900 \\ \mathrm{H} & 2.41491300 & -1.43660100 & -0.35834800 \\ \mathrm{C} & -2.39321800 & -1.15885500 & 0.18386300 \\ \mathrm{H} & -3.36055200 & -0.71209400 & 0.41189100 \\ \mathrm{H} & -2.29366500 & -1.23166500 & -0.90003500 \\ \mathrm{H} & -2.34718900 & -2.16336300 & 0.61817100 \\ & -1.21349600 & 2.09938100 & -0.83772500\end{array}$



$\mathrm{H}$
$-1.44239800 \quad 2.97572400 \quad-0.51405800$
$\mathrm{H}$
$-1.52896700 \quad 1.45267400 \quad-0.17509800$

Structure of TS3 with the Displacement Vectors Corresponding to Imaginary Frequency

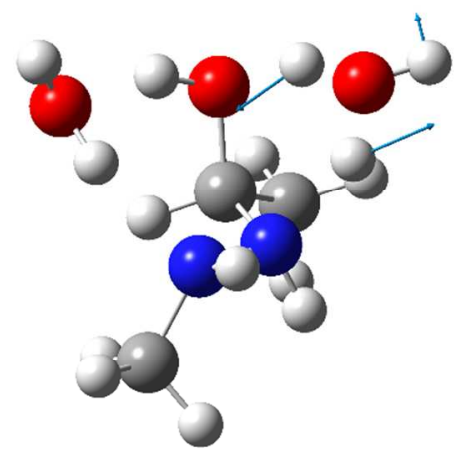

PC3-RC4 (Product Complex for Step 3-Reactant Complex for Step 4)

$\mathrm{C}$

$\begin{array}{lll}0.52511400 & -0.04996600 & -0.97734200\end{array}$

N $\quad 0.19118500 \quad-0.94502200 \quad 0.21727800$

$\mathrm{N} \quad-1.11565600 \quad-0.68906200 \quad 0.75164700$

$\mathrm{H} \quad 0.90027300 \quad-0.69135200 \quad 0.94673600$

$\mathrm{H} \quad-1.07927300 \quad-0.96276100 \quad 1.73006500$

$\mathrm{H} \quad-0.33099000 \quad-0.12353700 \quad-1.65139500$

O $\quad 0.70520400 \quad 1.23217700 \quad-0.50188700$

$\mathrm{H} \quad-0.16729500 \quad 1.67170100 \quad-0.42128000$

$\mathrm{H} \quad 0.31133500 \quad-1.93510900 \quad-0.03293800$

O $\quad 1.92451000 \quad 0.30147400 \quad 1.88535600$

$\mathrm{H} \quad 2.00885500 \quad 1.09808000 \quad 1.34576900$

$\mathrm{H} \quad 2.79830800 \quad 0.10459900 \quad 2.23656300$

C $\quad 1.79719800 \quad-0.56999400 \quad-1.60347700$ 


$\begin{array}{llll}\mathrm{H} & 1.67263000 & -1.59068000 & -1.96606400 \\ \mathrm{H} & 2.04923200 & 0.07113800 & -2.44681400 \\ \mathrm{H} & 2.61400600 & -0.53494600 & -0.87927300 \\ \mathrm{C} & -2.13801500 & -1.47532900 & 0.04939100 \\ \mathrm{H} & -3.08152900 & -1.30614800 & 0.56553100 \\ \mathrm{H} & -2.24006400 & -1.11781600 & -0.97473800 \\ \mathrm{H} & -1.91560600 & -2.54690700 & 0.04712400 \\ \mathrm{O} & -1.87296200 & 1.94418200 & -0.21883000 \\ \mathrm{H} & -2.25173900 & 2.76590200 & 0.10765300 \\ \mathrm{H} & -1.97664100 & 1.28548600 & 0.48203800\end{array}$

\section{TS4 (Transition State for Step 4)}

$\begin{array}{llll}\mathrm{C} & 0.77987500 & -0.76978800 & -0.84021600 \\ \mathrm{~N} & -0.29867400 & -0.58071600 & 0.88595500 \\ \mathrm{~N} & -1.54309700 & 0.10545700 & 0.67898200 \\ \mathrm{H} & 0.33878700 & 0.04128800 & 1.39924300 \\ \mathrm{H} & -1.83413200 & 0.54270300 & 1.55323600 \\ \mathrm{H} & -0.08686000 & -1.18582600 & -1.36459700 \\ \mathrm{O} & 1.13159900 & 0.41403800 & -1.11401900 \\ \mathrm{H} & 0.04589800 & 1.16891600 & -1.33544900 \\ \mathrm{H} & -0.45242300 & -1.42870400 & 1.43527200 \\ \mathrm{O} & 1.71702300 & 1.37164100 & 1.43513900 \\ \mathrm{H} & 1.98158900 & 1.29041000 & 0.50797400 \\ \mathrm{H} & 2.52070300 & 1.52639200 & 1.93899600 \\ \mathrm{C} & 1.80149800 & -1.74556800 & -0.35111900 \\ \mathrm{H} & 1.33874400 & -2.65095000 & 0.03757500 \\ \mathrm{H} & 2.42180100 & -2.01412000 & -1.21095500 \\ \mathrm{H} & 2.43983100 & -1.28360800 & 0.40289200\end{array}$




$$
\begin{array}{llrl}
\mathrm{C} & -2.59072000 & -0.82899600 & 0.23015000 \\
\mathrm{H} & -3.51435400 & -0.26460800 & 0.11762100 \\
\mathrm{H} & -2.30690700 & -1.24890800 & -0.73340700 \\
\mathrm{H} & -2.73592700 & -1.63148300 & 0.95552000 \\
\mathrm{O} & -0.96601900 & 1.58302200 & -1.25055500 \\
\mathrm{H} & -0.95131500 & 2.54007800 & -1.12650000 \\
\mathrm{H} & -1.31778100 & 1.04173700 & -0.32939800
\end{array}
$$

\section{Structure of TS4 with the Displacement Vectors Corresponding to Imaginary Frequency}

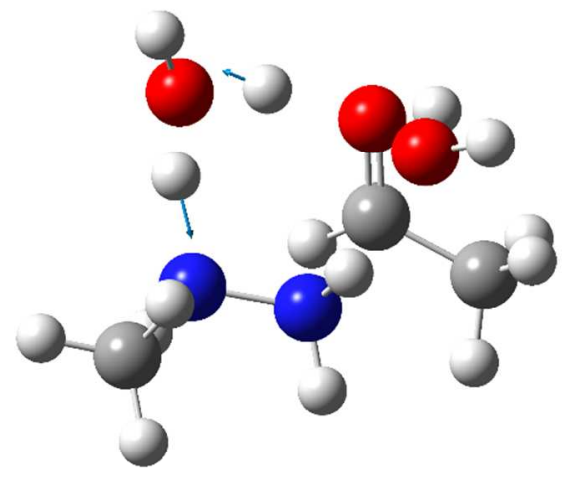

\section{PC4 (Product Complex for Step 4)}

$\begin{array}{llll}\mathrm{C} & 1.80868200 & -0.25371400 & -0.88545500 \\ \mathrm{~N} & -0.55313000 & -1.26103200 & 0.63852400 \\ \mathrm{~N} & -1.71309600 & -0.44435400 & 0.41502900 \\ \mathrm{H} & -0.05668700 & -0.81829400 & 1.41296800 \\ \mathrm{H} & -2.27960200 & -0.33176400 & 1.26077800 \\ \mathrm{H} & 1.34460300 & -0.64713200 & -1.80437800 \\ \mathrm{O} & 1.60360500 & 0.89899300 & -0.55869800\end{array}$




$\begin{array}{lrrr}\mathrm{H} & 0.00138600 & 1.72053300 & -1.09169300 \\ \mathrm{H} & -0.88295000 & -2.17333000 & 0.94694200 \\ \mathrm{O} & 0.21892500 & 1.14944800 & 1.86196000 \\ \mathrm{H} & 0.96670700 & 1.38706600 & 1.29732000 \\ \mathrm{H} & 0.32954000 & 1.64059600 & 2.68058900 \\ \mathrm{C} & 2.68105700 & -1.18934700 & -0.12493200 \\ \mathrm{H} & 2.13370300 & -2.11663300 & 0.05865900 \\ \mathrm{H} & 3.54215400 & -1.44441200 & -0.75002600 \\ \mathrm{H} & 3.01614000 & -0.74294200 & 0.81000400 \\ \mathrm{C} & -2.53691800 & -0.98012800 & -0.69534300 \\ \mathrm{H} & -3.36207700 & -0.29260600 & -0.86407800 \\ \mathrm{H} & -1.89830600 & -1.03743800 & -1.57291100 \\ \mathrm{H} & -2.90896300 & -1.96378200 & -0.41839400 \\ \mathrm{O} & -0.96982600 & 1.72211300 & -1.08883200 \\ \mathrm{H} & -1.24125900 & 2.63309700 & -0.94278400 \\ \mathrm{H} & -1.37936100 & 0.49944600 & 0.12107300\end{array}$

\section{Pathway 2 - One $\mathrm{H}_{2} \mathrm{O}$ Molecule as Reactant}

\section{$\underline{\text { RC1 (Reactant Complex for Step 1) }}$}

$\begin{array}{llll}\mathrm{C} & -0.57608600 & -0.62284400 & -0.39185700 \\ \mathrm{H} & -0.62619600 & -0.16284300 & -1.37240600 \\ \mathrm{~N} & 0.43928500 & -0.30521300 & 0.31721300 \\ \mathrm{~N} & 1.39583400 & 0.60766700 & -0.15391200 \\ \mathrm{H} & 1.31392400 & 1.43690000 & 0.43025900 \\ \mathrm{H} & 0.56057200 & -0.70593300 & 1.25094300 \\ \mathrm{O} & -2.06810800 & 1.49411400 & 0.06354600 \\ \mathrm{H} & -2.93997900 & 1.70754400 & -0.28279700 \\ \mathrm{H} & -1.68199900 & 2.34337100 & 0.29872900\end{array}$




$\begin{array}{llll}\mathrm{C} & 2.74565000 & 0.03909600 & -0.04760900 \\ \mathrm{H} & 3.44404400 & 0.81628200 & -0.35254100 \\ \mathrm{H} & 2.99214800 & -0.28774800 & 0.96753400 \\ \mathrm{H} & 2.82821400 & -0.80160000 & -0.73492300 \\ \mathrm{C} & -1.60037900 & -1.57840100 & 0.05909300 \\ \mathrm{H} & -2.57455200 & -1.09411200 & -0.02489000 \\ \mathrm{H} & -1.59776700 & -2.42874900 & -0.62759400 \\ \mathrm{H} & -1.43448800 & -1.92031100 & 1.07844800\end{array}$

\section{$\underline{\text { TS1 (Transition State for Step 1) }}$}

$\begin{array}{lrrr}\mathrm{C} & 0.82846600 & -0.18035400 & 0.33387300 \\ \mathrm{H} & 0.54880600 & -0.09337300 & 1.38193600 \\ \mathrm{~N} & -0.22752400 & -0.59928200 & -0.45375400 \\ \mathrm{~N} & -1.34056100 & 0.29978600 & -0.36087100 \\ \mathrm{H} & -1.65878100 & 0.48540000 & -1.30722300 \\ \mathrm{H} & 0.02595300 & -0.81901600 & -1.41138800 \\ \mathrm{O} & 1.00829500 & 1.39028200 & -0.04541000 \\ \mathrm{H} & 1.46924500 & 1.91920200 & 0.62727200 \\ \mathrm{H} & 0.05634600 & 1.66822400 & -0.08791800 \\ \mathrm{C} & -2.43607800 & -0.28917100 & 0.41710700 \\ \mathrm{H} & -3.26430100 & 0.41893300 & 0.41829800 \\ \mathrm{H} & -2.76618400 & -1.24441900 & 0.00146400 \\ \mathrm{H} & -2.10380600 & -0.43688800 & 1.44440700 \\ \mathrm{C} & 2.14330300 & -0.84264800 & 0.07316300 \\ \mathrm{H} & 2.93207800 & -0.36237200 & 0.64968000 \\ \mathrm{H} & 2.06713900 & -1.88273200 & 0.39251900 \\ \mathrm{H} & 2.38959800 & -0.80569800 & -0.98824700\end{array}$


Structure of TS1 with Displacement Vectors with Full Scale Corresponding to Imaginary Frequency

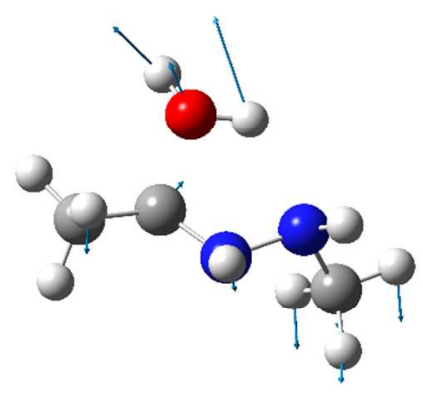

PC1-RC2 (Product Complex for Step 1-Reactant Complex for Step 2)

$\begin{array}{lrrr}\mathrm{C} & 0.82316200 & 0.04801500 & 0.32146700 \\ \mathrm{H} & 0.49301800 & 0.12305800 & 1.36024000 \\ \mathrm{~N} & -0.12984700 & -0.78910400 & -0.40168000 \\ \mathrm{~N} & -1.37765000 & -0.09082900 & -0.57676300 \\ \mathrm{H} & -1.91249600 & -0.59013900 & -1.29085000 \\ \mathrm{H} & 0.22839600 & -0.96082400 & -1.34109700 \\ \mathrm{O} & 0.79644900 & 1.32925800 & -0.28845200 \\ \mathrm{H} & 1.10182100 & 1.99968600 & 0.33153700 \\ \mathrm{H} & -1.19795000 & 0.86385200 & -0.92039400 \\ \mathrm{C} & -2.17844400 & -0.03678900 & 0.67858800 \\ \mathrm{H} & -3.15479200 & 0.37043500 & 0.42854000 \\ \mathrm{H} & -2.25833700 & -1.04962500 & 1.06230200 \\ \mathrm{H} & -1.67466800 & 0.61355900 & 1.38741100 \\ \mathrm{C} & 2.18603600 & -0.60579600 & 0.24312900\end{array}$




$$
\begin{array}{llll}
\mathrm{H} & 2.90527700 & -0.00028900 & 0.79409300 \\
\mathrm{H} & 2.15505500 & -1.60283600 & 0.68310000 \\
\mathrm{H} & 2.51103800 & -0.67399000 & -0.79727500
\end{array}
$$

\section{TS2 (Transition State for Step 1)}

$\begin{array}{llll}\mathrm{C} & -0.87450100 & -0.00421100 & -0.33810600 \\ \mathrm{~N} & 0.16980200 & -0.46423700 & 0.54051000 \\ \mathrm{~N} & 1.39197400 & 0.22688900 & 0.48377300 \\ \mathrm{H} & 1.82317700 & 0.21739600 & 1.41257000 \\ \mathrm{H} & -0.55744500 & -0.07731600 & -1.38253900 \\ \mathrm{O} & -1.08077800 & 1.38280600 & -0.14383900 \\ \mathrm{H} & 0.10572900 & -1.22069700 & 1.20105300 \\ \mathrm{H} & -1.51238000 & 1.51587200 & 0.70987100 \\ \mathrm{C} & -2.11323500 & -0.83981100 & -0.09681900 \\ \mathrm{H} & -1.91676200 & -1.88988500 & -0.31536500 \\ \mathrm{H} & -2.90997800 & -0.48695800 & -0.74992100 \\ \mathrm{H} & -2.43674100 & -0.74500300 & 0.94255800 \\ \mathrm{C} & 2.36005600 & -0.28025600 & -0.53794300 \\ \mathrm{H} & 3.24523700 & 0.35099700 & -0.50957800 \\ \mathrm{H} & 1.87882600 & -0.22593100 & -1.51059900 \\ \mathrm{H} & 2.60007300 & -1.30785300 & -0.28109400 \\ \mathrm{H} & 1.16013200 & 1.21403800 & 0.30098700\end{array}$

Structure of TS2 with the Displacement Vectors with Full Scale Corresponding to Imaginary Frequency 


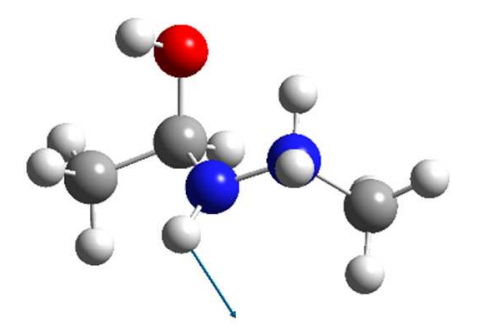

PC2-RC3 (Product Complex for Step 2-Reactant Complex for Step 3)

$\mathrm{N} \quad 0.12383400 \quad-0.50260800 \quad 0.67764300$

$\mathrm{N} \quad 1.38502100 \quad 0.17317500 \quad 0.53214400$

$\begin{array}{llll}\mathrm{H} & 1.88481000 & 0.07041700 & 1.41898200\end{array}$

$\mathrm{H} \quad-0.47593600 \quad-0.13637900-1.34389200$

$\begin{array}{llll}\mathrm{O} & -0.94958000 & 1.37972100 & -0.14109300\end{array}$

$\begin{array}{llll}\mathrm{H} & 0.29718700 & -1.50037400 & 0.57010700\end{array}$

$\mathrm{H} \quad-1.46055700 \quad 1.55300800 \quad 0.66006700$

C $\quad-2.14285200 \quad-0.75488500 \quad-0.12291600$

$\mathrm{H} \quad-2.00640200 \quad-1.82323200 \quad-0.29717000$

$\mathrm{H} \quad-2.87910000 \quad-0.37935900 \quad-0.83291700$

$\mathrm{H} \quad-2.51035000 \quad-0.60514800 \quad 0.89443900$

C $\quad 2.26072400 \quad-0.24712000 \quad-0.60297300$

$\begin{array}{llll}\mathrm{H} & 3.17105400 & 0.34560400 & -0.55612200\end{array}$

$\mathrm{H} \quad 1.73773500 \quad-0.06086700 \quad-1.53609400$

$\mathrm{H} \quad 2.48415500 \quad-1.30412700 \quad-0.48303100$

$\begin{array}{llll}\mathrm{H} & 1.14364600 & 1.17065100 & 0.45180300\end{array}$

$\underline{\text { TS3 (Transition State for Step 3) }}$ 


$\begin{array}{llll}\mathrm{C} & -1.02905300 & 0.01504200 & 0.38278400 \\ \mathrm{~N} & 0.20423300 & -0.49082700 & -0.56033700 \\ \mathrm{~N} & 1.51643700 & -0.60713900 & 0.00473200 \\ \mathrm{H} & 2.07694700 & -1.22323400 & -0.59621400 \\ \mathrm{H} & -0.55644400 & 0.14970100 & 1.37035900 \\ \mathrm{O} & -1.74779200 & -1.09919700 & 0.21594500 \\ \mathrm{H} & 0.27017400 & -0.03927300 & -1.47717100 \\ \mathrm{H} & -0.53903800 & -1.39048600 & -0.49066200 \\ \mathrm{C} & -1.59465000 & 1.30861100 & -0.15591800 \\ \mathrm{H} & -0.87842800 & 2.12961600 & -0.09483300 \\ \mathrm{H} & -2.46485300 & 1.56201600 & 0.45201800 \\ \mathrm{H} & -1.92599800 & 1.17560100 & -1.18773300 \\ \mathrm{C} & 2.20131100 & 0.71087300 & 0.19820700 \\ \mathrm{H} & 3.16070200 & 0.51063700 & 0.66795200 \\ \mathrm{H} & 1.57458900 & 1.32221100 & 0.84159300 \\ \mathrm{H} & 2.33459800 & 1.16813900 & -0.77874700 \\ \mathrm{H} & 1.41974200 & -1.09274200 & 0.90467400\end{array}$

Structure of TS3 with the Displacement Vectors Corresponding to Imaginary Frequency

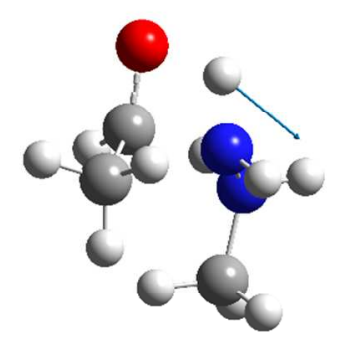

\section{PC3 (Product Complex for Step 3)}

$\mathrm{C}$

$-1.79858600-0.01005700-0.49458000$ 


$\begin{array}{llll}\mathrm{N} & 0.85865400 & -0.52892000 & 0.77711600 \\ \mathrm{~N} & 1.99623100 & -0.55251200 & -0.09985400 \\ \mathrm{H} & 2.77326200 & -1.11091100 & 0.27016900 \\ \mathrm{H} & -1.14952700 & 0.15600200 & -1.37388100 \\ \mathrm{O} & -2.27043000 & -1.10390100 & -0.29381200 \\ \mathrm{H} & 1.17776200 & -0.14240500 & 1.66380100 \\ \mathrm{H} & 0.58585000 & -1.49496000 & 0.94755700 \\ \mathrm{C} & -2.04933200 & 1.19092800 & 0.36011600 \\ \mathrm{H} & -1.09221800 & 1.62972100 & 0.65022900 \\ \mathrm{H} & -2.58200800 & 1.93799500 & -0.23559600 \\ \mathrm{H} & -2.63429300 & 0.92932000 & 1.24059600 \\ \mathrm{C} & 2.46373800 & 0.83138600 & -0.39743500 \\ \mathrm{H} & 3.27146000 & 0.76397300 & -1.12148700 \\ \mathrm{H} & 1.61769100 & 1.38250200 & -0.79822700 \\ \mathrm{H} & 2.81641300 & 1.27571800 & 0.53023700 \\ \mathrm{H} & 1.69994300 & -0.99925700 & -0.97233700\end{array}$

\section{Pathway 2 - Two $\mathrm{H}_{2} \mathrm{O}$ Molecules as Reactant}

\section{$\underline{\text { RC1 (Reactant Complex for Step 1) }}$}

$\begin{array}{llll}\mathrm{C} & 0.46000700 & -0.94732900 & -0.37050500 \\ \mathrm{~N} & -0.52630100 & -0.87732200 & 0.44258500 \\ \mathrm{~N} & -1.41586700 & 0.21275200 & 0.42739400 \\ \mathrm{H} & -1.54153900 & 0.48721600 & 1.39980600 \\ \mathrm{H} & 0.55926000 & -0.12494900 & -1.07143100 \\ \mathrm{O} & 2.30607500 & 0.77059500 & 0.44526200 \\ \mathrm{H} & 1.76358800 & 1.54096800 & 0.20590700 \\ \mathrm{H} & -0.69502100 & -1.62987500 & 1.11305700 \\ \mathrm{H} & 2.66924100 & 0.96159900 & 1.31404000\end{array}$




$\begin{array}{llll}\mathrm{C} & 1.38177200 & -2.09328900 & -0.41318400 \\ \mathrm{H} & 1.28134300 & -2.57214000 & -1.39100100 \\ \mathrm{H} & 2.39693000 & -1.70083500 & -0.33987100 \\ \mathrm{H} & 1.19013300 & -2.81651700 & 0.37689200 \\ \mathrm{C} & -2.71252800 & -0.17764900 & -0.15820700 \\ \mathrm{H} & -3.36920200 & 0.68666700 & -0.07779000 \\ \mathrm{H} & -2.56133600 & -0.41438600 & -1.21016800 \\ \mathrm{H} & -3.16442400 & -1.02947000 & 0.35584800 \\ \mathrm{O} & 0.27568300 & 2.45286400 & -0.39849900 \\ \mathrm{H} & -0.48650000 & 1.86668300 & -0.26279100 \\ \mathrm{H} & 0.12313300 & 2.91896200 & -1.22507200\end{array}$

\section{TS1 (Transition State for Step 1)}

$\begin{array}{llll}\mathrm{C} & 0.89675800 & -0.45798400 & -0.30728500 \\ \mathrm{~N} & -0.17888700 & -0.89003100 & 0.38260500 \\ \mathrm{~N} & -1.38764200 & -0.17071400 & 0.31253700 \\ \mathrm{H} & -1.62742200 & 0.12256700 & 1.25682000 \\ \mathrm{H} & 0.63618000 & 0.01331800 & -1.25158900 \\ \mathrm{O} & 1.54798600 & 0.96119200 & 0.37247300 \\ \mathrm{H} & 0.84870900 & 1.68907600 & 0.19722300 \\ \mathrm{H} & -0.05060500 & -1.52832400 & 1.16004100 \\ \mathrm{H} & 1.68811100 & 0.86994600 & 1.32730600 \\ \mathrm{C} & 2.07421600 & -1.38618700 & -0.33537000 \\ \mathrm{H} & 1.81282400 & -2.24980200 & -0.94790800 \\ \mathrm{H} & 2.93434500 & -0.88602700 & -0.77356600 \\ \mathrm{H} & 2.32578100 & -1.73067000 & 0.66888700 \\ \mathrm{C} & -2.47031700 & -0.99678300 & -0.23746200 \\ \mathrm{H} & -3.38834600 & -0.41031600 & -0.20794200\end{array}$




$\begin{array}{llll}\mathrm{H} & -2.23444500 & -1.23623600 & -1.27373900 \\ \mathrm{H} & -2.61167500 & -1.92293400 & 0.32696100 \\ \mathrm{O} & -0.41056900 & 2.43037900 & -0.21440400 \\ \mathrm{H} & -1.04171300 & 1.68712000 & -0.28940300 \\ \mathrm{H} & -0.42931800 & 2.92065600 & -1.04293300\end{array}$

Structure of TS1 with Displacement Vectors with Full Scale Corresponding to Imaginary Frequency

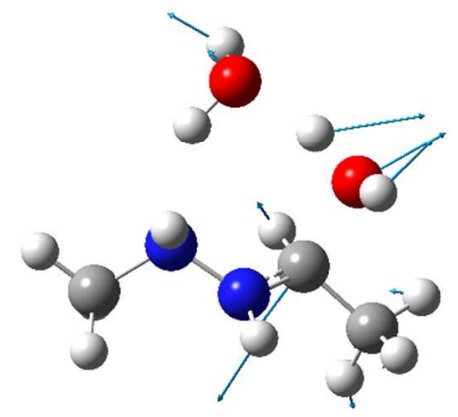

PC1-RC2 (Product Complex for Step 1-Reactant Complex for Step 2)

$\begin{array}{llll}\mathrm{C} & 0.99017400 & -0.32139600 & -0.30337900 \\ \mathrm{~N} & -0.09020300 & -0.97039800 & 0.30318700 \\ \mathrm{~N} & -1.32897500 & -0.27557500 & 0.31252000 \\ \mathrm{H} & -1.55572300 & -0.04236100 & 1.27694500\end{array}$




\begin{tabular}{|c|c|c|c|}
\hline $\mathrm{H}$ & 0.71443400 & 0.01843600 & -1.30106600 \\
\hline $\mathrm{O}$ & 1.33810600 & 1.02204500 & 0.34539900 \\
\hline $\mathrm{H}$ & 0.51158500 & 1.73561600 & 0.14652100 \\
\hline $\mathrm{H}$ & 0.12060200 & -1.45996800 & 1.16644500 \\
\hline $\mathrm{H}$ & 1.54534700 & 0.93546800 & 1.28912200 \\
\hline $\mathrm{C}$ & 2.24884800 & -1.15069700 & -0.28635500 \\
\hline $\mathrm{H}$ & 2.08140000 & -2.05979900 & -0.86237400 \\
\hline $\mathrm{H}$ & 3.06901400 & -0.59028400 & -0.73074300 \\
\hline $\mathrm{H}$ & 2.51616700 & -1.42647300 & 0.73638400 \\
\hline $\mathrm{C}$ & -2.40038600 & -1.11175300 & -0.24341800 \\
\hline $\mathrm{H}$ & -3.33915800 & -0.56382600 & -0.15836100 \\
\hline $\mathrm{H}$ & -2.18869600 & -1.29514200 & -1.29601300 \\
\hline $\mathrm{H}$ & -2.48682500 & -2.06756900 & 0.28052900 \\
\hline $\mathrm{O}$ & -0.61985100 & 2.31927700 & -0.17192400 \\
\hline $\mathrm{H}$ & -1.18518900 & 1.50926200 & -0.23903100 \\
\hline $\mathrm{H}$ & -0.64656600 & 2.80095400 & -1.00719300 \\
\hline
\end{tabular}

\section{$\underline{\text { TS2 (Transition State for Step 1) }}$}

$\begin{array}{lrrr}\mathrm{C} & 0.99662100 & -0.30635700 & -0.30185700 \\ \mathrm{~N} & -0.07827300 & -0.96880800 & 0.31106200 \\ \mathrm{~N} & -1.32448200 & -0.28296100 & 0.32308000 \\ \mathrm{H} & -1.55966800 & -0.06881400 & 1.28990800 \\ \mathrm{H} & 0.71438500 & 0.01299900 & -1.30474000 \\ \mathrm{O} & 1.32595200 & 1.03732600 & 0.32874800 \\ \mathrm{H} & 0.46754300 & 1.75142100 & 0.12854900 \\ \mathrm{H} & 0.14221200 & -1.43425700 & 1.18507900 \\ \mathrm{H} & 1.55370400 & 0.95846400 & 1.26806500 \\ \mathrm{C} & 2.26099800 & -1.12801000 & -0.28178900\end{array}$




$\begin{array}{lrrr}\mathrm{H} & 2.09936100 & -2.04337100 & -0.84935400 \\ \mathrm{H} & 3.07633000 & -0.56533800 & -0.73237700 \\ \mathrm{H} & 2.53266900 & -1.39298500 & 0.74279700 \\ \mathrm{C} & -2.38483400 & -1.11993800 & -0.25374900 \\ \mathrm{H} & -3.32904800 & -0.58163500 & -0.16750200 \\ \mathrm{H} & -2.16340300 & -1.28556500 & -1.30723300 \\ \mathrm{H} & -2.46529500 & -2.08378900 & 0.25570600 \\ \mathrm{O} & -0.65059600 & 2.28928400 & -0.16666900 \\ \mathrm{H} & -1.19340000 & 1.45506200 & -0.20857800 \\ \mathrm{H} & -0.69567000 & 2.75313700 & -1.01157900\end{array}$

Structure of TS2 with the Displacement Vectors with Full Scale Corresponding to Imaginary Frequency

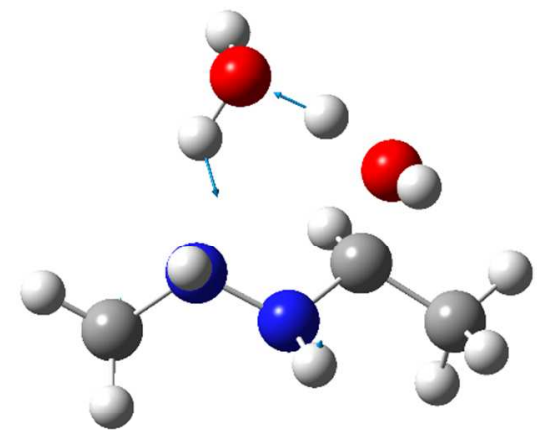

PC2-RC3 (Product Complex for Step 2-Reactant Complex for Step 3)

$\mathrm{C}$

$1.09270900-0.01984500-0.26205200$

$\mathrm{N} \quad 0.19102800 \quad-1.06904000 \quad 0.20451500$

$\mathrm{N} \quad-1.13363600 \quad-0.56389200 \quad 0.46135300$

$\mathrm{H} \quad-1.34054900 \quad-0.59178900 \quad 1.46234100$ 


$\begin{array}{llll}\mathrm{H} & 0.68828300 & 0.35215700 & -1.20570100 \\ \mathrm{O} & 1.11044800 & 1.10710500 & 0.59771000 \\ \mathrm{H} & -0.39815800 & 2.40631400 & -0.06812100 \\ \mathrm{H} & 0.53546700 & -1.49480500 & 1.06174000 \\ \mathrm{H} & 1.69600100 & 0.94354400 & 1.34697600 \\ \mathrm{C} & 2.46523900 & -0.62607800 & -0.46439800 \\ \mathrm{H} & 2.41589700 & -1.44251400 & -1.18391700 \\ \mathrm{H} & 3.14398300 & 0.13955900 & -0.83822900 \\ \mathrm{H} & 2.85800300 & -1.01233700 & 0.48050000 \\ \mathrm{C} & -2.16313400 & -1.33187500 & -0.28896000 \\ \mathrm{H} & -3.13731700 & -0.90239400 & -0.06212300 \\ \mathrm{H} & -1.93508000 & -1.23755000 & -1.34736900 \\ \mathrm{H} & -2.11247700 & -2.37112100 & 0.02338100 \\ \mathrm{O} & -1.29220600 & 2.09405300 & -0.26073700 \\ \mathrm{H} & -1.19401500 & 0.44846400 & 0.18635300 \\ \mathrm{H} & -1.53659200 & 2.45052400 & -1.12022100\end{array}$

\section{TS3 (Transition State for Step 3)}

$\begin{array}{llll}\mathrm{C} & -1.06792700 & -0.03278000 & 0.26432100 \\ \mathrm{~N} & -0.18365700 & -0.83872300 & -0.52048500 \\ \mathrm{~N} & 1.20055500 & -0.57961700 & -0.49808700 \\ \mathrm{H} & 1.57157500 & -0.66077600 & -1.44779900 \\ \mathrm{H} & -0.63736900 & 0.12416800 & 1.25763500 \\ \mathrm{O} & -1.20652100 & 1.28978100 & -0.25379900 \\ \mathrm{H} & 0.43400600 & 2.25638400 & 0.11097500 \\ \mathrm{H} & -0.48266000 & -1.59219900 & -1.11806000 \\ \mathrm{H} & -1.64313000 & 1.24822700 & -1.11356200 \\ \mathrm{C} & -2.40722000 & -0.73646100 & 0.36625600\end{array}$




$\begin{array}{llll}\mathrm{H} & -2.29725300 & -1.69680600 & 0.86935000 \\ \mathrm{H} & -3.09551800 & -0.11092300 & 0.93225400 \\ \mathrm{H} & -2.82405600 & -0.90136700 & -0.63100900 \\ \mathrm{C} & 1.96978000 & -1.45738300 & 0.43036900 \\ \mathrm{H} & 3.01680500 & -1.16364500 & 0.38658600 \\ \mathrm{H} & 1.56982900 & -1.31047100 & 1.43064900 \\ \mathrm{H} & 1.84079900 & -2.48840100 & 0.11252700 \\ \mathrm{O} & 1.38203700 & 2.05369000 & 0.15569300 \\ \mathrm{H} & 1.34548100 & 0.43201300 & -0.22943700 \\ \mathrm{H} & 1.71127700 & 2.40415700 & 0.98907500\end{array}$

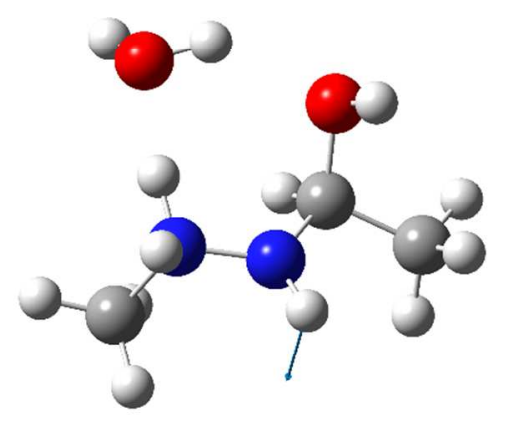

PC3-RC4 (Product Complex for Step 3-Reactant Complex for Step 4)

C $\quad-1.07492700 \quad 0.08797900 \quad 0.27107500$

$\mathrm{N} \quad-0.41210100 \quad-0.79391600 \quad-0.69518100$ 


\begin{tabular}{|c|c|c|c|}
\hline $\mathrm{N}$ & 1.01757900 & -0.78450900 & -0.55466900 \\
\hline $\mathrm{H}$ & 1.40928700 & -1.08406900 & -1.45006400 \\
\hline $\mathrm{H}$ & -0.63046400 & 0.01137900 & 1.26824600 \\
\hline $\mathrm{O}$ & -0.85579900 & 1.42512600 & -0.11623200 \\
\hline $\mathrm{H}$ & 1.00867200 & 2.15475400 & 0.25197100 \\
\hline $\mathrm{H}$ & -0.72218000 & -1.75725600 & -0.59057800 \\
\hline $\mathrm{H}$ & -1.29824900 & 1.58280600 & -0.96005900 \\
\hline $\mathrm{C}$ & -2.54442200 & -0.27746500 & 0.31267400 \\
\hline $\mathrm{H}$ & -2.68389900 & -1.28594700 & 0.70432900 \\
\hline $\mathrm{H}$ & -3.06362800 & 0.42431200 & 0.96388000 \\
\hline $\mathrm{H}$ & -2.97491900 & -0.22064900 & -0.68990000 \\
\hline $\mathrm{C}$ & 1.57703300 & -1.61543600 & 0.54843500 \\
\hline $\mathrm{H}$ & 2.65972700 & -1.50943700 & 0.52911000 \\
\hline $\mathrm{H}$ & 1.18303700 & -1.24987100 & 1.49317400 \\
\hline $\mathrm{H}$ & 1.29267000 & -2.65202400 & 0.38410700 \\
\hline $\mathrm{O}$ & 1.86440600 & 1.74139700 & 0.07104500 \\
\hline $\mathrm{H}$ & 1.33248700 & 0.20951800 & -0.41174000 \\
\hline $\mathrm{H}$ & 2.43414000 & 1.92281100 & 0.82486300 \\
\hline
\end{tabular}

\section{TS4 (Transition State for Step 4)}

$\begin{array}{llll}\mathrm{C} & 1.06416900 & 0.04804100 & -0.29336900 \\ \mathrm{~N} & 0.10661200 & -0.57948400 & 0.78330700 \\ \mathrm{~N} & -1.29143300 & -0.49030700 & 0.46671900 \\ \mathrm{H} & -1.81349300 & -0.55697800 & 1.34590600 \\ \mathrm{H} & 0.50434200 & 0.10706500 & -1.23662200 \\ \mathrm{O} & 1.21997600 & 1.23859000 & 0.36105800 \\ \mathrm{H} & -0.23869400 & 2.09301500 & -0.14771800 \\ \mathrm{H} & 0.33026000 & -1.50834500 & 1.15177800\end{array}$




$\begin{array}{llll}\mathrm{H} & 0.51847200 & 0.41992500 & 1.27734700 \\ \mathrm{C} & 2.29568200 & -0.81660900 & -0.41689500 \\ \mathrm{H} & 2.06266800 & -1.78584200 & -0.86063300 \\ \mathrm{H} & 3.00480800 & -0.29439900 & -1.05977300 \\ \mathrm{H} & 2.75831200 & -0.95497900 & 0.56273300 \\ \mathrm{C} & -1.75768400 & -1.52956900 & -0.49346700 \\ \mathrm{H} & -2.81672600 & -1.35400300 & -0.66556900 \\ \mathrm{H} & -1.19895800 & -1.42009300 & -1.41893900 \\ \mathrm{H} & -1.59790600 & -2.51081400 & -0.05393500 \\ \mathrm{O} & -1.21582400 & 2.02980500 & -0.28080300 \\ \mathrm{H} & -1.43472200 & 0.51132800 & 0.08468200 \\ \mathrm{H} & -1.43083600 & 2.38431400 & -1.14908800\end{array}$

Structure of TS4 with the Displacement Vectors Corresponding to Imaginary Frequency

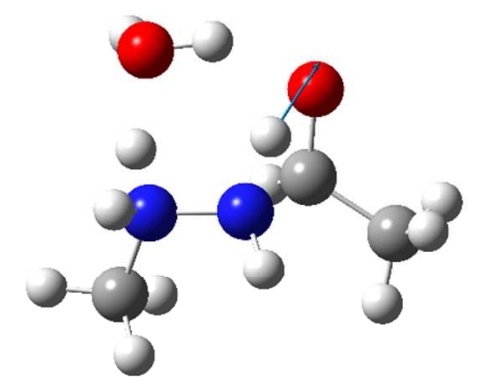

\section{PC4 (Product Complex for Step 4)}

$\begin{array}{llll}\text { C } & 1.80414400 & 0.02132600 & -0.42826900\end{array}$ 


\begin{tabular}{|c|c|c|c|}
\hline $\mathrm{N}$ & -0.71226300 & -0.99486700 & 0.85348500 \\
\hline $\mathrm{N}$ & -1.87889700 & -0.28775700 & 0.40407900 \\
\hline $\mathrm{H}$ & -2.64432800 & -0.31769500 & 1.08428100 \\
\hline $\mathrm{H}$ & 1.09378200 & -0.08390300 & -1.26527600 \\
\hline $\mathrm{O}$ & 1.82107700 & 1.04795100 & 0.21835400 \\
\hline $\mathrm{H}$ & 0.26229100 & 1.95495400 & 0.03607800 \\
\hline $\mathrm{H}$ & -0.98870700 & -1.95852300 & 1.03150700 \\
\hline $\mathrm{H}$ & -0.43970500 & -0.58990600 & 1.74727300 \\
\hline $\mathrm{C}$ & 2.69842200 & -1.14177700 & -0.17393900 \\
\hline $\mathrm{H}$ & 2.07375200 & -2.01431900 & 0.03687900 \\
\hline $\mathrm{H}$ & 3.26286200 & -1.36501200 & -1.08292700 \\
\hline $\mathrm{H}$ & 3.37230000 & -0.94506700 & 0.65755900 \\
\hline $\mathrm{C}$ & -2.33781400 & -0.80952500 & -0.90664500 \\
\hline $\mathrm{H}$ & -3.19929500 & -0.22442400 & -1.21892500 \\
\hline $\mathrm{H}$ & -1.51805900 & -0.69923800 & -1.61142300 \\
\hline $\mathrm{H}$ & -2.60991100 & -1.85626700 & -0.79328700 \\
\hline $\mathrm{O}$ & -0.70174300 & 2.10779900 & 0.03722100 \\
\hline $\mathrm{H}$ & -1.57264000 & 0.71140300 & 0.27852900 \\
\hline $\mathrm{H}$ & -0.89741100 & 2.70022100 & -0.69470500 \\
\hline
\end{tabular}

\section{Pathway 2 - Three $\mathrm{H}_{2} \mathrm{O}$ Molecules as Reactant}

\section{$\underline{\text { RC1 (Reactant Complex for Step 1) }}$}

$\begin{array}{llll}\mathrm{C} & -0.36865600 & -1.44995000 & -0.51698500 \\ \mathrm{~N} & -0.44551500 & -0.58693600 & 0.42254200 \\ \mathrm{~N} & -1.52117900 & 0.24035800 & 0.70620500 \\ \mathrm{H} & 1.84383700 & 0.99174500 & 2.25205400 \\ \mathrm{H} & -1.58746300 & 0.31211400 & 1.71822100 \\ \mathrm{H} & -1.24883800 & -1.62971300 & -1.12388500\end{array}$




\begin{tabular}{llll}
$\mathrm{O}$ & 1.98831700 & 0.75639100 & -1.16138900 \\
$\mathrm{H}$ & 1.26352600 & 1.39963100 & -1.09828500 \\
$\mathrm{H}$ & 0.39938700 & -0.37017500 & 0.98895000 \\
$\mathrm{O}$ & 1.89497700 & 0.18452400 & 1.72704400 \\
$\mathrm{H}$ & 2.27747800 & 0.63332500 & -0.24960300 \\
$\mathrm{H}$ & 2.42845700 & -0.43000500 & 2.24377500 \\
$\mathrm{C}$ & 0.88672600 & -2.18189700 & -0.79351700 \\
$\mathrm{H}$ & 0.67545800 & -3.25249900 & -0.81003400 \\
$\mathrm{H}$ & 1.24223500 & -1.89109100 & -1.78457500 \\
$\mathrm{H}$ & 1.65948100 & -1.96229800 & -0.05928200 \\
$\mathrm{C}$ & -2.78850800 & -0.15486300 & 0.09604500 \\
$\mathrm{H}$ & -3.55099900 & 0.49451400 & 0.51930500 \\
$\mathrm{H}$ & -2.74849000 & 0.01486300 & -0.98025400 \\
$\mathrm{H}$ & -3.04043000 & -1.19981300 & 0.30294700 \\
$\mathrm{O}$ & -0.27798200 & 2.40750700 & -0.80093200 \\
$\mathrm{H}$ & -0.22282700 & 3.31270500 & -0.48140700 \\
$\mathrm{H}$ & -0.84381900 & 1.93563200 & -0.17018800 \\
\hline & & &
\end{tabular}

\section{TS1 (Transition State for Step 1)}

$\begin{array}{llll}\mathrm{C} & 0.33983000 & -0.28519800 & -0.99802000 \\ \mathrm{~N} & -0.32669500 & -0.87642100 & -0.01656000 \\ \mathrm{~N} & -1.51422700 & -0.31078200 & 0.48238000 \\ \mathrm{H} & 1.81447700 & -0.20184200 & 2.63276600 \\ \mathrm{H} & -1.37266100 & -0.14163700 & 1.47594400 \\ \mathrm{H} & -0.26500000 & 0.35305500 & -1.63342200 \\ \mathrm{O} & 1.27676700 & 1.16956000 & -0.32466900 \\ \mathrm{H} & 0.57336200 & 1.83036900 & -0.12333300 \\ \mathrm{H} & 0.14014800 & -1.58344800 & 0.54459800\end{array}$




$\begin{array}{llll}\mathrm{O} & 2.13186400 & -0.33359000 & 1.73296200 \\ \mathrm{H} & 1.65584900 & 0.83240400 & 0.51739100 \\ \mathrm{H} & 3.06071600 & -0.57280700 & 1.82011800 \\ \mathrm{C} & 1.47696000 & -1.03587600 & -1.60921900 \\ \mathrm{H} & 1.06279000 & -1.82993800 & -2.23346300 \\ \mathrm{H} & 2.07522400 & -0.37192600 & -2.22723100 \\ \mathrm{H} & 2.10293100 & -1.48308600 & -0.83565300 \\ \mathrm{C} & -2.65679200 & -1.21502600 & 0.29200800 \\ \mathrm{H} & -3.52705800 & -0.75153300 & 0.75537800 \\ \mathrm{H} & -2.83909900 & -1.32716000 & -0.77595300 \\ \mathrm{H} & -2.48730500 & -2.19844900 & 0.73959700 \\ \mathrm{O} & -1.04843700 & 2.43752800 & -0.20594400 \\ \mathrm{H} & -1.40888400 & 3.15467400 & 0.32328300 \\ \mathrm{H} & -1.54057500 & 1.63036600 & 0.03182600\end{array}$

Structure of TS1 with Displacement Vectors with Full Scale Corresponding to Imaginary Frequency 


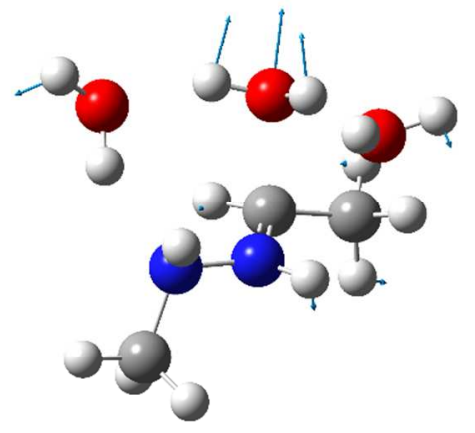

PC1-RC2 (Product Complex for Step 1-Reactant Complex for Step 2)

$\begin{array}{llll}\mathrm{C} & 0.42528800 & -0.49304400 & -0.89495700 \\ \mathrm{~N} & -0.41016100 & -0.99933300 & 0.12113900 \\ \mathrm{~N} & -1.49177900 & -0.15901600 & 0.50308400 \\ \mathrm{H} & 1.85712700 & 0.72498100 & 2.51133300 \\ \mathrm{H} & -1.29407500 & 0.22794800 & 1.42368500 \\ \mathrm{H} & -0.17277400 & -0.208666000 & -1.75996600 \\ \mathrm{O} & 1.08207700 & 0.81827000 & -0.52626200 \\ \mathrm{H} & 0.35982300 & 1.56805300 & -0.56346200 \\ \mathrm{H} & 0.09680500 & -1.38814700 & 0.91154400 \\ \mathrm{O} & 2.17793500 & 0.30898200 & 1.70304500 \\ \mathrm{H} & 1.54256800 & 0.73454700 & 0.37691200 \\ \mathrm{H} & 3.14023500 & 0.30689500 & 1.76085100 \\ \mathrm{C} & 1.53985100 & -1.45122700 & -1.23512600 \\ \mathrm{H} & 1.11312800 & -2.37359800 & -1.62663500 \\ \mathrm{H} & 2.19350800 & -1.01064700 & -1.98567400 \\ \mathrm{H} & 2.12639200 & -1.68421500 & -0.34319100 \\ \mathrm{C} & -2.74332500 & -0.92188400 & 0.56496500 \\ \mathrm{H} & -3.53062200 & -0.25731700 & 0.92213700\end{array}$




$\begin{array}{llll}\mathrm{H} & -2.99423000 & -1.26382100 & -0.43840400 \\ \mathrm{H} & -2.66721400 & -1.78566500 & 1.23193400 \\ \mathrm{O} & -0.91355800 & 2.25623700 & -0.76805400 \\ \mathrm{H} & -1.08093500 & 3.08547200 & -0.30768700 \\ \mathrm{H} & -1.47867500 & 1.56163600 & -0.36205300\end{array}$

\section{TS2 (Transition State for Step 1)}

\begin{tabular}{|c|c|c|c|}
\hline & 0.43921600 & -0.56686200 & -0.83023500 \\
\hline $\mathrm{N}$ & -0.32741800 & -0.94189100 & 0.31522000 \\
\hline $\mathrm{N}$ & -1.45080700 & -0.09302000 & 0.59713900 \\
\hline $\mathrm{H}$ & 1.93395000 & 1.14531800 & 2.32727200 \\
\hline $\mathrm{H}$ & -1.32753000 & 0.29052900 & 1.53200000 \\
\hline $\mathrm{H}$ & -0.22175800 & -0.50749500 & -1.69608500 \\
\hline $\mathrm{O}$ & 1.00745400 & 0.79406200 & -0.74549700 \\
\hline $\mathrm{H}$ & 0.08661800 & 1.56205800 & -0.82902500 \\
\hline $\mathrm{H}$ & 0.25575500 & -1.06265600 & 1.13854400 \\
\hline $\mathrm{O}$ & 2.18640500 & 0.56665600 & 1.59949600 \\
\hline $\mathrm{H}$ & 1.52894100 & 0.86474500 & 0.09640100 \\
\hline $\mathrm{H}$ & 3.13422200 & 0.42185500 & 1.69319400 \\
\hline $\mathrm{C}$ & 1.57246000 & -1.54288400 & -1.04799100 \\
\hline $\mathrm{H}$ & 1.16795700 & -2.53841800 & -1.22574200 \\
\hline $\mathrm{H}$ & 2.16499400 & -1.23533700 & -1.90817500 \\
\hline $\mathrm{H}$ & 2.21698800 & -1.57572000 & -0.16600800 \\
\hline $\mathrm{C}$ & -2.70102200 & -0.86727300 & 0.56016500 \\
\hline $\mathrm{H}$ & -3.52336700 & -0.20333700 & 0.82763800 \\
\hline $\mathrm{H}$ & -2.85170900 & -1.23269600 & -0.45484700 \\
\hline $\mathrm{H}$ & -2.66727900 & -1.71303200 & 1.24977300 \\
\hline 0 & -1.03402600 & 1.98814500 & -0.89060400 \\
\hline
\end{tabular}



$\mathrm{H}$
$-1.15727300 \quad 2.85495600-0.48461300$
$\mathrm{H}$
$\begin{array}{llll}-1.43551600 & 1.24482500 & -0.28563400\end{array}$

Structure of TS2 with the Displacement Vectors Corresponding to Imaginary Frequency

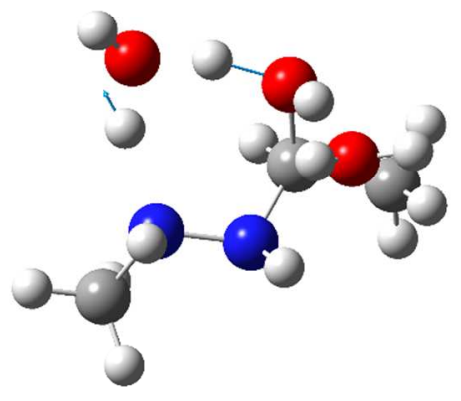

PC2-RC3 (Product Complex for Step 2-Reactant Complex for Step 3)

C

$$
0.46665200-0.32434600-0.88682900
$$

$\mathrm{N}$

$0.08723500 \quad-1.01598100 \quad 0.34820800$

$\mathrm{N}$

$\begin{array}{lll}-1.15440700 & -0.49536900 & 0.87147300\end{array}$

$\mathrm{H}$

$$
\begin{array}{lll}
2.25838400 & 1.12093000 & 2.25461900
\end{array}
$$

$\mathrm{H}$

$\begin{array}{lll}-1.11609500 & -0.54054600 & 1.89118800\end{array}$

$\mathrm{H}$

$$
\begin{array}{lll}
-0.35915600 & -0.42025000 & -1.59483900
\end{array}
$$

$\mathrm{O}$

$$
\begin{array}{llll}
0.63808400 & 1.06726600 & -0.69236900
\end{array}
$$

$\mathrm{H}$

$\begin{array}{lll}-1.09679500 & 2.10447500 & -0.73913100\end{array}$

$\mathrm{H}$

$\begin{array}{lll}0.80681300 & -0.82365200 & 1.04741300\end{array}$

$\mathrm{O}$

$$
\begin{array}{llll}
2.39794900 & 0.62306300 & 1.44196400
\end{array}
$$

$\mathrm{H}$

$\begin{array}{llll}1.38626200 & 1.19240100 & -0.08426500\end{array}$

$\mathrm{H}$

$\begin{array}{lll}3.34448300 & 0.44823100 & 1.41040500\end{array}$

C

$\begin{array}{llll}1.71698400 & -0.99017500 & -1.42710300\end{array}$

$\mathrm{H}$

$\begin{array}{llll}1.52297900 & -2.03814100 & -1.65314400\end{array}$

$\mathrm{H}$

$\begin{array}{llll}2.02861300 & -0.47730000 & -2.33627600\end{array}$ 


$\begin{array}{llll}\mathrm{H} & 2.52555700 & -0.92887100 & -0.69475500 \\ \mathrm{C} & -2.31958500 & -1.28347400 & 0.38984900 \\ \mathrm{H} & -3.21984600 & -0.84240500 & 0.81226500 \\ \mathrm{H} & -2.34671900 & -1.22034900 & -0.69480000 \\ \mathrm{H} & -2.19109300 & -2.31163900 & 0.71476400 \\ \mathrm{O} & -1.96826000 & 1.78960300 & -0.46115900 \\ \mathrm{H} & -2.49266500 & 2.56395000 & -0.23786900 \\ \mathrm{H} & -1.30699700 & 0.50113700 & 0.60366500\end{array}$

\section{TS3 (Transition State for Step 3)}

$\begin{array}{llll}\mathrm{C} & 0.35505500 & -0.46924200 & -0.79703400 \\ \mathrm{~N} & -0.28302400 & -0.83836800 & 0.43540400 \\ \mathrm{~N} & -1.47369500 & -0.19156300 & 0.81924800 \\ \mathrm{H} & 2.64933500 & 0.21667200 & 2.06732900 \\ \mathrm{H} & -1.44591700 & -0.00534900 & 1.82487100 \\ \mathrm{H} & -0.40337900 & -0.37690400 & -1.58108500 \\ \mathrm{O} & 0.95737200 & 0.81557300 & -0.73444000 \\ \mathrm{H} & -0.43650100 & 2.08630800 & -0.76161100 \\ \mathrm{H} & 0.06824300 & -1.53374300 & 1.07351000 \\ \mathrm{O} & 2.89846900 & 0.62927000 & 1.23440900 \\ \mathrm{H} & 1.66344200 & 0.79088400 & -0.06014000 \\ \mathrm{H} & 3.78332800 & 0.30520900 & 1.03964900 \\ \mathrm{C} & 1.36300600 & -1.54669000 & -1.15386700 \\ \mathrm{H} & 0.86528700 & -2.50318200 & -1.31303100 \\ \mathrm{H} & 1.88441000 & -1.25699500 & -2.06491500 \\ \mathrm{H} & 2.09790700 & -1.65251200 & -0.35163700 \\ \mathrm{C} & -2.71448400 & -0.94070900 & 0.46828700 \\ \mathrm{H} & -3.57163800 & -0.34728100 & 0.78071200\end{array}$




$\begin{array}{llll}\mathrm{H} & -2.71802400 & -1.08414400 & -0.60940200 \\ \mathrm{H} & -2.69436200 & -1.89715900 & 0.98334200 \\ \mathrm{O} & -1.39899800 & 2.11227200 & -0.62054700 \\ \mathrm{H} & -1.62311600 & 2.99323600 & -0.30644000 \\ \mathrm{H} & -1.49817800 & 0.75740500 & 0.35658500\end{array}$

Structure of TS3 with the Displacement Vectors Corresponding to Imaginary Frequency

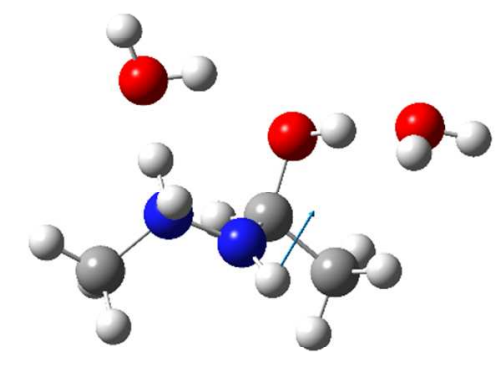

PC3-RC4 (Product Complex for Step 3-Reactant Complex for Step 4)

$\mathrm{N}$

$\mathrm{N}$

H

H

H

O

$\mathrm{H}$

H

O

H

$$
\begin{array}{ccc}
0.35512000 & -0.57912100 & -0.71984200 \\
-0.14994500 & -0.83467900 & 0.63860400 \\
-1.41378600 & -0.19229200 & 0.87866600 \\
2.69978900 & 0.35019400 & 1.86738500 \\
-1.49162200 & -0.04739000 & 1.88735800 \\
-0.44577900 & -0.63734400 & -1.46497600 \\
0.83137300 & 0.74020700 & -0.78241400 \\
-0.58572700 & 2.06873900 & -0.89790300 \\
-0.28586300 & -1.82998700 & 0.80003600 \\
2.86628300 & 0.86519000 & 1.07145200 \\
1.58262200 & 0.82412500 & -0.16094300
\end{array}
$$




$\begin{array}{llll}\mathrm{H} & 3.79073000 & 0.71266400 & 0.85203100 \\ \mathrm{C} & 1.43166600 & -1.60626700 & -1.01511100 \\ \mathrm{H} & 1.01579400 & -2.61449800 & -1.04382500 \\ \mathrm{H} & 1.87222700 & -1.38376000 & -1.98623300 \\ \mathrm{H} & 2.21128000 & -1.55825100 & -0.25202000 \\ \mathrm{C} & -2.61641700 & -0.91149500 & 0.37341400 \\ \mathrm{H} & -3.49101200 & -0.30882500 & 0.60929400 \\ \mathrm{H} & -2.52191800 & -1.03027500 & -0.70296100 \\ \mathrm{H} & -2.67556000 & -1.87902200 & 0.86693700 \\ \mathrm{O} & -1.52342100 & 2.06683700 & -0.64856600 \\ \mathrm{H} & -1.75395700 & 2.96358000 & -0.38811700 \\ \mathrm{H} & -1.39099200 & 0.76228400 & 0.43849600\end{array}$

$\underline{\text { TS4 (Transition State for Step 4) }}$

$\begin{array}{llll}\mathrm{C} & 0.68568100 & -0.44706500 & -0.75613700 \\ \mathrm{~N} & -0.00725700 & -0.69529300 & 0.64138400 \\ \mathrm{~N} & -1.36607900 & -0.21413900 & 0.67874800 \\ \mathrm{H} & 1.36404800 & 1.64699200 & 2.07860300 \\ \mathrm{H} & -1.59954600 & 0.00642900 & 1.65054200 \\ \mathrm{H} & -0.08472500 & -0.68668700 & -1.50088900 \\ \mathrm{O} & 1.06759300 & 0.84992100 & -0.80133200 \\ \mathrm{H} & -0.28384700 & 1.69054200 & -1.01518500 \\ \mathrm{H} & -0.00429300 & -1.68152700 & 0.90932500 \\ \mathrm{O} & 1.62760900 & 0.88545000 & 1.55001400 \\ \mathrm{H} & 1.55788100 & 1.10721500 & 0.50291600 \\ \mathrm{H} & 0.70607100 & 0.00014400 & 1.34534200 \\ \mathrm{C} & 1.82745300 & -1.44620000 & -0.81483200\end{array}$




$\begin{array}{lrrr}\mathrm{H} & 1.47248500 & -2.47756500 & -0.77472700 \\ \mathrm{H} & 2.34926600 & -1.29299600 & -1.75899100 \\ \mathrm{H} & 2.53208100 & -1.26627800 & 0.00071500 \\ \mathrm{C} & -2.36050000 & -1.17361600 & 0.12464900 \\ \mathrm{H} & -3.33396500 & -0.69178800 & 0.17973500 \\ \mathrm{H} & -2.10494400 & -1.38221800 & -0.91081300 \\ \mathrm{H} & -2.35159000 & -2.08334800 & 0.72080100 \\ \mathrm{O} & -1.27881700 & 1.81899300 & -0.92834100 \\ \mathrm{H} & -1.40319800 & 0.71866000 & 0.09921900 \\ \mathrm{H} & -1.44924800 & 2.72482000 & -0.65231600\end{array}$

Structure of TS4 with the Displacement Vectors Corresponding to Imaginary Frequency

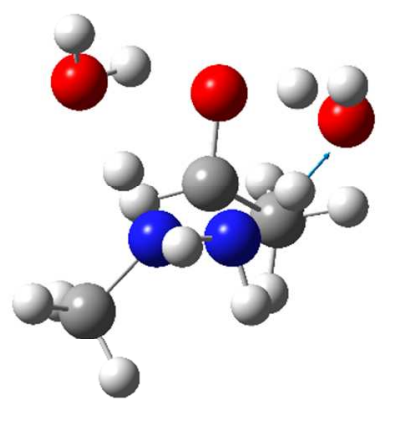

\section{PC4 (Product Complex for Step 4)}

$\begin{array}{llll}\mathrm{C} & 1.56663600 & 0.49659800 & 1.03633700 \\ \mathrm{~N} & -0.67903800 & 0.89502400 & -0.84890300 \\ \mathrm{~N} & -1.88120200 & 0.17979500 & -0.52997900 \\ \mathrm{H} & 1.04679000 & -1.93493100 & -2.35116700 \\ \mathrm{H} & -2.40865000 & -0.09639600 & -1.36303100\end{array}$




\begin{tabular}{llll}
$\mathrm{H}$ & 0.92026000 & 0.92000900 & 1.82184100 \\
$\mathrm{O}$ & 1.57598200 & -0.70617700 & 0.85554400 \\
$\mathrm{H}$ & -0.00299900 & -1.61369300 & 1.23439200 \\
$\mathrm{H}$ & -0.94875200 & 1.70777100 & -1.39848500 \\
$\mathrm{O}$ & 1.16818600 & -1.09410500 & -1.90128600 \\
$\mathrm{H}$ & 1.56312700 & -1.29978200 & -1.04257000 \\
$\mathrm{H}$ & -0.11112000 & 0.27650700 & -1.43746600 \\
$\mathrm{C}$ & 2.40282600 & 1.45775600 & 0.26719800 \\
$\mathrm{H}$ & 1.76601900 & 2.26101600 & -0.10980800 \\
$\mathrm{H}$ & 3.12267200 & 1.91367100 & 0.95381800 \\
$\mathrm{H}$ & 2.92634800 & 0.96062300 & -0.54796100 \\
$\mathrm{C}$ & -2.74362400 & 0.96818700 & 0.38376100 \\
$\mathrm{H}$ & -3.61473500 & 0.36675100 & 0.63301400 \\
$\mathrm{H}$ & -2.15770800 & 1.19118700 & 1.27189500 \\
$\mathrm{H}$ & -3.04379000 & 1.88435200 & -0.11968700 \\
$\mathrm{O}$ & -0.96526900 & -1.72905800 & 1.15449500 \\
$\mathrm{H}$ & -1.58561700 & -0.68612900 & -0.01760400 \\
$\mathrm{H}$ & -1.13638500 & -2.67521500 & 1.13119600 \\
\hline & & & \\
$\mathrm{H}$ & & &
\end{tabular}

